\title{
MEET-REDUCIBLE SUBMAXIMAL CLONES DETERMINED BY NONTRIVIAL EQUIVALENCE RELATIONS
}

\section{LUC E. F. DIÉKOUAM ${ }^{1}$, YANNICK L. T. JEUFACK ${ }^{2}$, ÉTIENNE R.}

\section{A. TEMGOUA ${ }^{3}$ and MARCEL TONGA ${ }^{4}$}

1Department of Mathematics

Ecole Normale Supérieure

University of Maroua

P. O. Box 55 Maroua

Cameroon

e-mail: lucdiekouam@yahoo.fr

${ }^{2,4}$ Department of Mathematics

Faculty of Sciences

University of Yaoundé-1

P. O. Box 812 Yaoundé

Cameroon

e-mail: tenkius2000@yahoo.fr

tongamarcel@yahoo.fr

${ }^{3}$ Department of Mathematics

Ecole Normale Supérieure

University of Yaoundé-1

P. O. Box 47 Yaoundé

Cameroon

e-mail: retemgoua@yahoo.fr

2010 Mathematics Subject Classification: Primary 08A40; Secondary 08A02, 18B35.

Keywords and phrases: clones, submaximal, equivalence relations, maximal.

Received March 20, 2017 


\begin{abstract}
The structure of the lattice of clones on a finite set has been proven to be very complex. To better understand the top of this lattice, it is important to provide a characterization of submaximal clones in the lattice of clones. It is known that the clones $\operatorname{Pol}(\theta)$ and $\operatorname{Pol}(\rho)$ (where $\theta$ is a nontrivial equivalence relation on $E_{k}=\{0, \ldots, k-1\}$, and $\rho$ belongs to one of the six types of relations which characterize maximal clones) are maximal clones. In this paper, we provide a classification of relations (of Rosenberg's list) $\rho$ on $E_{k}$ for which the clone $\operatorname{Pol}(\theta) \cap \operatorname{Pol}(\rho)$ is maximal in $\operatorname{Pol}(\theta)$.
\end{abstract}

\title{
1. Introduction
}

The structure of the lattice of clones on a finite set of more than two elements is quite complex. For instance, its cardinality is $2 \aleph_{0}$. For a better comprehension of some intervals in this lattice, it is important to provide a characterization of maximal and submaximal clones. Maximal clones have been investigated extensively by I. G. Rosenberg, and a complete characterization of these can be found in [14]. More precisely, it is proved that for a given nontrivial equivalence relation $\theta$ on a finite set and a central relation $\rho$ on the same set, the clones $\operatorname{Pol}(\theta)$ and $\operatorname{Pol}(\rho)$ are maximal. For a unary central relation on an arbitrary finite set, Rosenberg and Szendrei [16, 17] investigated the submaximal clones of their polymorphisms and obtain new results on those of prime permutations on a finite set. Submaximal clones for a set with two and three elements were completely described and classified in [7, 11, 12]. However, for sets with more than three elements, only partial results on their submaximal clones are found in the literature (see, e.g., [7]). Recently, Temgoua and Rosenberg obtained a characterization of all binary central relations for which the clone $\operatorname{Pol}(\theta) \cap \operatorname{Pol}(\rho)$ is maximal in $\operatorname{Pol}(\theta)$, given any nontrivial equivalence relation $\theta$ and a binary central relation $\rho$ [18]. 
In this paper, we characterize the relations $\rho$ for which the clones of the form $\operatorname{Pol}(\theta) \cap \operatorname{Pol}(\rho)$ is maximal in $\operatorname{Pol}(\theta)$, where $\theta$ is a nontrivial equivalence relation on a given finite set.

The rest of the paper is organized as follows: Section 2 is devoted to basic definitions and notations needed for the clarity of our presentation. In Section 3, we present the non-submaximality when $\rho$ is a partial order or a prime affine relation. Section 4 is devoted to the characterization of type of equivalence relations or prime permutation relations which give submaximality. In Section 5, we characterize the relations $\rho$ (resp., central relations or $h$-regularly generated relations) for which $\operatorname{Pol}(\theta) \cap \operatorname{Pol}(\rho)$ is maximal in $\operatorname{Pol}(\theta)$. Section 6 concludes the paper.

\section{Preliminaries}

In this section, we recall some of the main definitions and notations. Readers needing more background on the topic may refer to [7]. Let $E_{k}=\{0,1, \ldots, k-1\}$ be a finite set of $k$ elements with $k \geq 1$. Let $n, s \in \mathbb{N}^{*}$. An $n$-ary operation on $E_{k}$ is a function from $E_{k}^{n}$ to $E_{k}$. The set of all $n$-ary operations on $E_{k}$ is denoted by $\mathcal{O}^{n}\left(E_{k}\right)$ and we set

$\mathcal{O}\left(E_{k}\right)=\bigcup_{0<n<\omega} \mathcal{O}^{n}\left(E_{k}\right)$. For $1 \leq i \leq s$, the $s$-ary $i$-th projection $e_{i}^{s}$ is defined as $e_{i}^{s}\left(x_{1}, \ldots, x_{s}\right)=x_{i}$ for all $x_{1}, \ldots, x_{s}$ in $E_{k}$. For $f \in \mathcal{O}^{n}\left(E_{k}\right)$ and $g_{1}, \ldots, g_{n} \in \mathcal{O}^{m}\left(E_{k}\right)$, we define their composition to be the $m$-ary operation $f\left[g_{1}, \ldots, g_{n}\right]$ defined by:

$$
f\left[g_{1}, \ldots, g_{n}\right]\left(x_{1}, \ldots, x_{m}\right)=f\left(g_{1}\left(x_{1}, \ldots, x_{m}\right), \ldots, g_{n}\left(x_{1}, \ldots, x_{m}\right)\right) .
$$

A clone on $E_{k}$ is a subset $F$ of $\mathcal{O}\left(E_{k}\right)$ which contains all the projections and is closed under composition. It is known that the intersection of an 
arbitrary set of clones on $E_{k}$ is a clone on $E_{k}$. Thus for $F \subseteq \mathcal{O}\left(E_{k}\right)$, there exists a smallest clone containing $F$, called the clone generated by $F$ and denoted by $\langle F\rangle .\langle F\rangle$ is also the set of term operations of the nonindexed algebra $\mathcal{A}=(A ; F)$, with $A=E_{k}$. The clones on $E_{k}$, ordered by inclusion, form a complete lattice denoted by $\mathcal{L}\left(E_{k}\right)$. A clone $C \in \mathcal{L}\left(E_{k}\right)$ is called maximal if it is covered only by $\mathcal{O}\left(E_{k}\right)$. A clone $C \in \mathcal{L}\left(E_{k}\right)$ is called submaximal if it is covered only by a maximal clone.

Let $h$ be a positive integer. An $h$-ary relation $\rho$ is a subset of $E_{k}^{h}$. For $\rho \subseteq E_{k}^{2}$, we write $a \rho b$ for $(a, b) \in \rho$. An $h$-ary relation $\rho$ is called totally symmetric if for every permutation $\sigma$ of $\{1, \ldots, h\}$ and each $h$-tuple $\left(a_{1}, \ldots, a_{h}\right) \in E_{k}^{h}$,

$$
\left(a_{1}, \ldots, a_{h}\right) \in \rho \text { if }\left(a_{\sigma(1)}, \ldots, a_{\sigma(h)}\right) \in \rho .
$$

$\tau_{h}^{E_{k}}$ is the $h$-ary relation defined by $\left(a_{1}, \ldots, a_{h}\right) \in \tau_{h}^{E_{k}}$ if there exist $i, j \in\{1, \ldots, h\}$ such that $i \neq j$ and $a_{i}=a_{j}$. An $h$-ary relation $\rho$ is called totally reflexive if $\tau_{h}^{E_{k}} \subseteq \rho$. For $h=2$, the concepts totally reflexive and totally symmetric coincide with the usual notions of reflexive and symmetric. If $\rho$ is totally reflexive and totally symmetric, we define the center of $\rho$ denoted by $C_{\rho}$ as follows:

$$
C_{\rho}=\left\{a \in E_{k}:\left(a, a_{2}, \ldots, a_{h}\right) \in \rho \text { for all } a_{2}, \ldots, a_{h} \in E_{k}\right\} .
$$

Let $\theta$ be a binary relation and $m \in \mathbb{N}^{*}$; for $\mathbf{a}=\left(a_{1}, \ldots, a_{m}\right) \in E_{k}^{m}$ and $\mathbf{b}=\left(b_{1}, \ldots, b_{m}\right) \in E_{k}^{m}$, we write $\mathbf{a} \theta \mathbf{b}$ if $\left(a_{i}, b_{i}\right) \in \theta$ for $1 \leq i \leq m$. Assume that $\theta$ is an equivalence relation on $E_{k}$. The $\theta$-class of $a \in E_{k}$ will be denoted by $[a]_{\theta}$. 
A permutation $\pi$ on $E_{k}$ is prime if all cycles of $\pi$ have the same prime length.

A subset $\sigma \subseteq E_{k}^{4}$ is called affine if there is a binary operation + on $E_{k}$ such that $\left(E_{k},+\right)$ is an abelian group and $(a, b, c, d) \in \sigma \Leftrightarrow a+b$ $=c+d$. An affine relation $\sigma$ is prime if $\left(E_{k},+\right)$ is an abelian $p$-group for some prime $p$, that is, all elements of the group have the same prime order $p$.

An $h$-ary relation $\rho$ on $E_{k}$ is called central, if $\rho$ is a nonempty proper subset of $E_{k}$ or $\rho$ has the following three properties: $\rho$ is totally reflexive; $\rho$ is totally symmetric and $C_{\rho}$ is a nonempty proper subset of $E_{k}$.

For $h \geq 3$, a family $T=\left\{\mathcal{V}_{1} ; \cdots ; \mathcal{V}_{m}\right\}$ of equivalence relations on $E_{k}$ is called $h$-regular if each $\mathcal{V}_{i}$ has exactly $h$ equivalence classes and $\bigcap\left\{B_{i} \mid 1 \leq i \leq m\right\}$ is nonempty for arbitrary equivalence classes $B_{i}$ of $\mathcal{V}_{i}$, $1 \leq i \leq m$. For $3 \leq h \leq k$, an $h$-regular (or $h$-regularly generated) relation on $E_{k}$ determined by the $h$-regular family $T$ (often denoted by $\lambda_{T}$ ), consists of all $h$-tuples whose set of components meets at most $h-1$ classes of each $\mathcal{V}_{i}(1 \leq i \leq m)$.

Let $f \in \mathcal{O}^{n}\left(E_{k}\right)$ and $\rho$ be an $h$-ary relation on $E_{k}$. The operation $f$ preserves $\rho$ if for all $\left(a_{1, i}, \ldots, a_{h, i}\right) \in \rho(i=1, \ldots, n)$, we have

$$
\left(f\left(a_{1,1}, \ldots, a_{1, n}\right), f\left(a_{2,1}, \ldots, a_{2, n}\right), \ldots, f\left(a_{h, 1}, \ldots, a_{h, n}\right)\right) \in \rho
$$

The set of operations on $E_{k}$ preserving $\rho$ is a clone denoted by $\operatorname{Pol}(\rho)$. The maximal clones have been described for $k=2$ (respectively, $k=3$ and $k \geq 4$ ) in Post [11] (respectively, Jablonskij [5] and Rosenberg $[14,15])$. They are of the form $\operatorname{Pol}(\rho)$ where $\rho$ belongs to one of six families of relations which include some familiar and easily defined 
relations. For clones $C$ and $D$ on $E_{k}$, we say that $C$ is maximal in $D$ if $D$ covers $C$ in $\mathcal{L}\left(E_{k}\right)$; submaximal clones have already been define on page 2. All submaximal clones are known for $k=2$ (see [11]) and $k=3$ (see [7]).

For $n \geq 3$, an $n$-ary operation $f$ is called a near-unanimity operation provided that $f(y, x, \ldots, x) \approx f(x, y, \ldots, x) \approx \ldots \approx f(x, x, \ldots, y) \approx x$ for all $x, y \in E_{k}$. We recall the following Baker-Pixley Theorem which will be useful in the proofs:

Theorem 2.1 ([1]). Let $\mathcal{A}=(A, F)$ be a finite algebra which contains $a$ "near unanimity function" of arity $d+1((d+1)$-ary near-unanimity term or nu-term). Then, an operation $f: A^{n} \rightarrow A$ is term function for $\mathcal{A}$ iff each subuniverse of $\mathcal{A}^{d}$ is preserved by $f$.

\section{Partial Order, Prime Affine Relations}

In this section, we prove that the clones $\operatorname{Pol}(\theta) \cap \operatorname{Pol}(\rho)$ is not maximal in $\operatorname{Pol}(\theta)$ in case $\theta$ is a nontrivial equivalence relation on $E_{k}$ and $\rho$ is either a partial order with least and greatest elements or a prime affine relation on $E_{k}$.

Theorem 3.1. If $\theta$ is a nontrivial equivalence relation and $\rho$ is a partial order with least and greatest elements, on a finite set $E_{k}$, then $\operatorname{Pol}(\theta) \cap \operatorname{Pol}(\rho)$ is not submaximal in $\operatorname{Pol}(\theta)$.

Proof. $\theta$ and $\rho$ are incomparable. In fact, $\theta \nsubseteq \rho$ because $\theta$ is a nontrivial symmetric relation and $\rho$ is an antisymmetric relation. We also have $\rho \nsubseteq \theta$, otherwise since $\rho$ is a partial order with least and greatest element and $\theta$ is a transitive relation, $\theta$ will be trivial (equal to $\left.E_{k}^{2}\right)$; this is a contradiction. Without lost of generality, we can consider that the least element of $\rho$ is 0 and the greatest element of $\rho$ is 1 . 
Let $\rho^{\prime}$ and $r$ be the relations defined by: $\rho^{\prime}:=\theta \circ \rho \circ \theta$ and $r:=\rho \cap \theta$.

If $\rho^{\prime} \neq E_{k}^{2}$, we have $\operatorname{Pol}(\theta) \cap \operatorname{Pol}(\rho) \subsetneq \operatorname{Pol}\left(\rho^{\prime}\right) \subsetneq \operatorname{Pol}(\theta)$.

Suppose that $\rho^{\prime}=E_{k}^{2}$, then $(1,0) \in E_{k}^{2}=\rho^{\prime}$ and it follows that $(1,0) \in \theta$ or there is $b \in E_{k} \backslash\{0\}$ such that $(b, 0) \in \theta$. Therefore $r \neq \Delta_{E_{k}}=\left\{(a, a): a \in E_{k}\right\}$.

Since $r$ and $r^{-1}$ are subset of $\theta, r \circ r^{-1}$ is a subset of $\theta$.

If $r \circ r^{-1} \neq \theta$, then $\operatorname{Pol}(\theta) \cap \operatorname{Pol}(\rho) \subsetneq \operatorname{Pol}(\theta) \cap \operatorname{Pol}\left(r \circ r^{-1}\right) \subsetneq \operatorname{Pol}(\theta)$.

If $r \circ r^{-1}=\theta$, then $\operatorname{Pol}(r) \subsetneq \operatorname{Pol}(\theta)$ and it can be proved that each equivalence class of $\theta$ contains a least and a greatest element. It follows from Theorem 3.3 of [19] that $\operatorname{Pol}(r)$ is a meet-irreducible maximal subclone of $\operatorname{Pol}(\theta)$. Using the fact that $\operatorname{Pol}(\theta) \cap \operatorname{Pol}(\rho) \subsetneq \operatorname{Pol}(r)$, we conclude that $\operatorname{Pol}(\theta) \cap \operatorname{Pol}(\rho)$ is not maximal in $\operatorname{Pol}(\theta)$.

For a prime affine relation, there is no meet-reducible submaximality in the set of polymorphisms of a nontrivial equivalence relation. This is proved by the following theorem.

Theorem 3.2. Let $\alpha$ be a prime affine relation and $\theta$ be a nontrivial equivalence relation. We have

$$
\operatorname{Pol}(\theta) \cap \operatorname{Pol}(\alpha) \subsetneq \operatorname{Pol}\left(\alpha_{1}\right) \subsetneq \operatorname{Pol}(\theta),
$$

where

$$
\alpha_{1}=\{(a, b, c, d) \in \alpha:(a, b),(a, c),(a, d) \in \theta\} .
$$


Proof. Let $(a, b) \in \theta$ such that $a \neq b$, then the binary operation $g_{1}$ defined on $E_{k}$ by:

$$
g_{1}(x, y)= \begin{cases}a & \text { if }(x, y) \in\{(a, a) ;(a, b) ;(b, a)\} \\ b & \text { otherwise }\end{cases}
$$

preserves $\theta$ and does not preserve $\alpha_{1}$. Therefore $\operatorname{Pol}\left(\alpha_{1}\right) \subsetneq \operatorname{Pol}(\theta)$.

Also it is easy to see that $\operatorname{Pol}(\theta) \cap \operatorname{Pol}(\alpha) \subseteq \operatorname{Pol}\left(\alpha_{1}\right)$. Now, let $(a, b) \notin \theta$ and let $g_{2}$ be a ternary operation on $E_{k}$ defined by:

$$
g_{2}(x, y, z)= \begin{cases}b \quad \text { if }(x, y, z) \theta(a, a, b) \\ a \quad \text { otherwise }\end{cases}
$$

$g_{2}$ preserves $\alpha_{1}$ and does not preserve $\alpha$; therefore $\operatorname{Pol}(\theta) \cap \operatorname{Pol}(\alpha) \subsetneq \operatorname{Pol}\left(\alpha_{1}\right)$.

\section{Equivalence Relations, Prime Permutation Relations}

Let $k>1, \theta$ a nontrivial equivalence relation on $E_{k}$ with blocks (equivalence classes) $B_{0}, \ldots, B_{t_{1}-1}$ (where $\left.2 \leq t_{1} \leq k\right)$ and $\rho \quad$ a nontrivial equivalence relation distinct from $\theta$ with blocks $C_{0}, \ldots, C_{t_{2}-1}$ (where $2 \leq t_{2} \leq k$ ). In this section, we determine the meet-reducible clones of the form $\operatorname{Pol}(\theta) \cap \operatorname{Pol}(\rho)$ maximal in $\operatorname{Pol}(\theta)$, where $\theta$ and $\rho$ are two distinct nontrivial equivalence relations on $E_{k}$.

We define $\mu: E_{k} \rightarrow E_{t_{1}}$ by $\mu(x)=i$ if $x \in B_{i}$ and $\nu: E_{k} \rightarrow E_{t_{2}}$ by $\nu(x)=i$ if $x \in C_{i}$. Set $D=\operatorname{Pol}(\theta) \cap \operatorname{Pol}(\rho), \nabla_{E_{k}}=E_{k}^{2}$ and $\gamma=\theta \cap \rho$. Clearly $\gamma$ is an equivalence relation on $E_{k}$ and $D \subseteq \operatorname{Pol}(\theta) \cap \operatorname{Pol}(\gamma)$.

Theorem 4.1. Let $\theta$ and $\rho$ be two distinct nontrivial equivalence relations on a finite set $E_{k} . \operatorname{Pol}(\theta) \cap \operatorname{Pol}(\rho)$ is submaximal in $\operatorname{Pol}(\theta)$ if and only if $\theta$ and $\rho$ satisfy one of the following statement: 
(a) $\theta \subsetneq \rho$ or $\rho \subsetneq \theta$;

(b) $\rho \cap \theta=\Delta_{E_{k}}$ and $\rho \circ \theta=\nabla_{E_{k}}$.

To prove this result, we need the following remark and lemmas.

Remark 4.2. In the condition (b), it follows from $\rho \circ \theta=\nabla_{E_{k}}$ that there exist $u_{i} \in B_{i}\left(i=0, \ldots, t_{1}-1\right)$ such that $\left(u_{p}, u_{q}\right) \in \rho$ for all $0 \leq p, q \leq t_{1}-1$. Also $\rho \circ \theta=\nabla_{E_{k}}$ is equivalent to $\theta \circ \rho=\nabla_{E_{k}}$.

Lemma 4.3. Let $\beta$ be a binary relation on $E_{k}$, $\rho$ and $\theta$ satisfying the condition (a) or (b) of Theorem 4.1.

$$
\text { If } \operatorname{Pol}(\theta) \cap \operatorname{Pol}(\rho) \subseteq \operatorname{Pol}(\beta) \text {, then } \beta \in\left\{\Delta_{E_{k}} ; \rho ; \theta ; \nabla_{E_{k}}\right\} \text {. }
$$

\section{Proof.}

- Assume that $\rho$ and $\theta$ satisfy condition (a). Moreover, suppose that $\rho \subsetneq \theta, \operatorname{Pol}(\theta) \cap \operatorname{Pol}(\rho) \subseteq \operatorname{Pol}(\beta)$ and $\beta \neq \Delta_{E_{k}}$. For any distinct $a_{1}, a_{2} \in E_{k}$ and for any $b_{i} \in\left[a_{i}\right]_{\rho}(i=1,2)$, the function $f: E_{k} \rightarrow E_{k}$ defined by $f\left(a_{i}\right)=b_{i},(i=1,2)$ and $f(x)=x$ for all $x \in E_{k} \backslash\left\{a_{1} ; a_{2}\right\}$ satisfies $f \in \operatorname{Pol}(\theta) \cap \operatorname{Pol}(\rho)$. Since $\operatorname{Pol}(\theta) \cap \operatorname{Pol}(\rho) \subseteq \operatorname{Pol}(\beta), f \in \operatorname{Pol}(\beta)$. It follows that $\left[a_{1}\right]_{\rho} \times\left[a_{2}\right]_{\rho} \subseteq \beta$ whenever $\left(a_{1}, a_{2}\right) \in \beta$. In the following, we denote by $\beta / \rho$ the relation on $E_{k} / \rho:=\left\{[a]_{\rho} \mid a \in E_{k}\right\}$ defined by: $\left(\left[a_{1}\right]_{\rho},\left[a_{2}\right]_{\rho}\right)$ $\in \beta / \rho \Leftrightarrow\left(a_{1}, a_{2}\right) \in \beta$. Similarly, we define $\rho / \rho$ and $\theta / \rho$ and we have $\operatorname{Pol}(\theta / \rho) \cap \operatorname{Pol}(\rho / \rho) \subseteq \operatorname{Pol}(\beta / \rho)$. Since $\rho / \rho=\Delta_{E_{k} / \rho}, \operatorname{Pol}(\theta / \rho) \cap \operatorname{Pol}(\rho / \rho)$ $=\operatorname{Pol}(\theta / \rho)$ is the maximal clone on $E_{k} / \rho$ determined by the nontrivial equivalence relation $\theta / \rho$ and it follows that $\beta / \rho \in\left\{\rho / \rho=\Delta_{E_{k} / \rho} ; \theta / \rho\right.$; $\left.\nabla_{E_{k} / \rho}=\nabla_{E_{k}} / \rho\right\}$. Hence $\beta \in\left\{\rho ; \theta ; \nabla_{E_{k}}\right\}$. 
- Assume that $\rho$ and $\theta$ satisfy condition (b). In this case the function $\varphi: E_{k} \rightarrow E_{k} / \rho \times E_{k} / \theta$, defined by $a \mapsto\left([a]_{\rho},[a]_{\theta}\right)$ is a bijection. This bijection gives a decomposition of $E_{k}$ into a Cartesian product of $E_{k} / \rho$ and $E_{k} / \theta$ and one deduces that the operations in $\operatorname{Pol}(\theta) \cap \operatorname{Pol}(\rho)$ correspond to the operations that act coordinatewise on $E_{k} / \rho \times E_{k} / \theta$.

Lemma 4.4. Let $\beta$ be a binary relation on $E_{k}$. If $\rho$ and $\theta$ satisfy condition (a) or (b) in Theorem 4.1, then Pol $(\theta) \cap \operatorname{Pol}(\rho)$ contains a majority operation.

\section{Proof.}

- Assume that $\rho$ and $\theta$ satisfy condition (a). In addition, assume that $\rho \subsetneq \theta$. In each set $B_{i}$, we fix an element $v_{B_{i}}\left(i=0, \ldots, t_{1}-1\right)$. We consider the majority operation $m$ defined on $E_{k}$ by:

$$
m\left(x_{1}, x_{2}, x_{3}\right)= \begin{cases}x_{i} \quad & \text { if }\left(x_{i}, x_{j}\right) \in \rho \text { and } x_{l} \notin\left[x_{i}\right]_{\rho} \\ & \text { for some } 1 \leq i<j \leq 3, \text { such that } l \notin\{i ; j\}, \\ x_{1} & \text { if }\left\{x_{1} ; x_{2}\right\} \subseteq\left[x_{3}\right]_{\rho}, \\ v_{\left[x_{l}\right]_{\theta}} & \text { if } x_{i} \notin\left[x_{j}\right]_{\rho} \text { for } 1 \leq i<j \leq 3 \text { and } \\ & \left(x_{l}, x_{m}\right) \in \theta \text { for some } 1 \leq l<m \leq 3, \\ 0 & \text { otherwise. }\end{cases}
$$

Let us show that $m \in \operatorname{Pol}(\rho) \cap \operatorname{Pol}(\theta)$.

Let $\left(a_{i}, b_{i}\right) \in \rho, 1 \leq i \leq 3$;

- if $\left\{a_{1} ; a_{2}\right\} \subseteq\left[a_{3}\right]_{\rho}$, then $\left\{b_{1} ; b_{2}\right\} \subseteq\left[b_{3}\right]_{\rho}$ and $m\left(a_{1}, a_{2}, a_{3}\right)=a_{1} \rho b_{1}$ $=m\left(b_{1}, b_{2}, b_{3}\right)$, 
- otherwise, if there exist $1 \leq i<j \leq 3$ such that $\left(a_{i}, a_{j}\right) \in \rho$, $l \notin\{i ; j\}$ and $a_{l} \notin\left[a_{i}\right]_{\rho}$, then $\left(b_{i}, b_{j}\right) \in \rho, l \notin\{i ; j\}$ and $b_{l} \notin\left[b_{i}\right]_{\rho}$, hence $m\left(a_{1}, a_{2}, a_{3}\right)=a_{i} \rho b_{i}=m\left(b_{1}, b_{2}, b_{3}\right)$,

- otherwise, if there exist $1 \leq l<m \leq 3$ such that $\left(a_{l}, a_{m}\right) \in \theta$, then with transitivity of $\theta$ and the fact that $\rho \subsetneq \theta$ we have $\left(b_{l}, b_{m}\right) \in \theta$ and $m\left(a_{1}, a_{2}, a_{3}\right)=v_{\left[a_{l}\right]_{\theta}}=v_{\left[b_{l}\right]_{\theta}}=m\left(b_{1}, b_{2}, b_{3}\right)$,

- otherwise, we have $m\left(a_{1}, a_{2}, a_{3}\right)=0=m\left(b_{1}, b_{2}, b_{3}\right)$. Therefore $m \in \operatorname{Pol}(\rho)$.

Let $\left(a_{i}, b_{i}\right) \in \theta, 1 \leq i \leq 3$;

- if $\left\{a_{1} ; a_{2}\right\} \subseteq\left[a_{3}\right]_{\rho}$ then the transitivity of $\theta$ and the fact that $\rho \subsetneq \theta$, we have $\left\{a_{1} ; a_{2}\right\} \subseteq\left[a_{3}\right]_{\theta}$ and $\left\{b_{1} ; b_{2}\right\} \subseteq\left[b_{3}\right]_{\theta}$; therefore

$$
\left(m\left(a_{1}, a_{2}, a_{3}\right), m\left(b_{1}, b_{2}, b_{3}\right)\right) \subseteq\left\{a_{i} ; b_{i} ; v_{\left[a_{i}\right]_{\theta}} ; v_{\left[b_{l}\right]_{\theta}}\right\}^{2} \subseteq \theta,
$$

- otherwise, if there exist $1 \leq i<j \leq 3$ such that $\left(a_{i}, a_{j}\right) \in \rho$, $l \notin\{i ; j\}$ and $a_{l} \notin\left[a_{i}\right]_{\rho}$, then $\left(b_{i}, b_{j}\right) \in \theta$; it follows that $m\left(b_{1}, b_{2}, b_{3}\right) \neq 0$ and $m\left(b_{1}, b_{2}, b_{3}\right) \in\left[m\left(a_{1}, a_{2}, a_{3}\right)\right]_{\theta}$. Since $l \notin\{i ; j\}$ and $b_{l} \notin\left[b_{i}\right]_{\rho}$,

$$
m\left(a_{1}, a_{2}, a_{3}\right)=a_{i} \rho b_{i}=m\left(b_{1}, b_{2}, b_{3}\right),
$$

- otherwise, if there exist $1 \leq l<m \leq 3$ such that $\left(a_{l}, a_{m}\right) \in \theta$, then with transitivity of $\theta$, we have $\left(b_{l}, b_{m}\right) \in \theta$, and

$$
m\left(a_{1}, a_{2}, a_{3}\right)=v_{\left[a_{l}\right]_{\theta}}=v_{\left[b_{l}\right]_{\theta}}=m\left(b_{1}, b_{2}, b_{3}\right),
$$

- otherwise, we have $m\left(a_{1}, a_{2}, a_{3}\right)=0=m\left(b_{1}, b_{2}, b_{3}\right)$. Therefore $m \in \operatorname{Pol}(\theta)$.

- Assume that $\rho$ and $\theta$ satisfy condition (b). With the decomposition of $E_{k}$ into a Cartesian product of $E_{k} / \rho$ and $E_{k} / \theta$, we can say that, if $m_{1}$ is a majority operation on $E_{k} / \rho$ and $m_{2}$ is a majority operation on 
$E_{k} / \theta$, then the operation $m$ on $E_{k} / \rho \times E_{k} / \theta$ that acts like $m_{i}$ in the $i$-th coordinate $(i=1,2)$ is a majority operation on $E_{k} / \rho \times E_{k} / \theta$ that preserves $\rho$ and $\theta$.

Lemmas 4.3 and 4.4 together with Theorem 2.1 prove the if-direction of Theorem 4.1 and the only if-direction follows from the following lemmas.

Lemma 4.5. If $\Delta_{E_{k}} \subsetneq \gamma \subsetneq \theta$ and $\gamma \subsetneq \rho$, then $D \subsetneq \operatorname{Pol}(\theta) \cap \operatorname{Pol}(\gamma) \subsetneq \operatorname{Pol}(\theta)$.

Proof. $\gamma$ is a nontrivial equivalence relation on $E_{k}$ distinct from $\theta$ and $\rho$. Thus $D \subseteq \operatorname{Pol}(\theta) \cap \operatorname{Pol}(\gamma) \subsetneq \operatorname{Pol}(\theta)$. Let us prove that $D \neq \operatorname{Pol}(\theta)$ $\cap \operatorname{Pol}(\gamma) ;$ let $(a, b) \in \theta \backslash \gamma$ and $(c, d) \in \rho \backslash \gamma$. Then $(a, b) \notin \rho$ and $(c, d) \notin \theta$. Define $f \in \mathcal{O}^{1}\left(E_{k}\right)$ by:

$$
f(x)= \begin{cases}a & \text { if } x \in B_{\mu(c)}, \\ b & \text { otherwise. }\end{cases}
$$

Since $a \theta b, f \in \operatorname{Pol}(\theta)$. In addition $\gamma \subseteq \theta$ and $f$ is constant on each block of $\theta$, hence $f \in \operatorname{Pol}(\gamma)$. Therefore $f \in \operatorname{Pol}(\theta) \cap \operatorname{Pol}(\gamma)$ while $f \notin D$ since $c \rho d$ and $(f(c), f(d))=(a, b) \notin \rho$.

Lemma 4.6. Let $\rho$ and $\theta$ be two nontrivial equivalence relations which are incomparable.

$$
\text { If } \rho \cap \theta \neq \Delta_{E_{k}}, \quad \text { then } \quad \operatorname{Pol}(\theta) \cap \operatorname{Pol}(\rho) \subsetneq \operatorname{Pol}(\theta) \cap \operatorname{Pol}(\rho \cap \theta) \subsetneq \operatorname{Pol}(\theta)
$$

and $\operatorname{Pol}(\theta) \cap \operatorname{Pol}(\rho \cap \theta)$ is maximal in $\operatorname{Pol}(\theta)$.

Proof. It follows from the assumptions and Lemma 4.5 that $\operatorname{Pol}(\theta) \cap \operatorname{Pol}(\rho) \subsetneq \operatorname{Pol}(\theta) \cap \operatorname{Pol}(\rho \cap \theta) \subsetneq \operatorname{Pol}(\theta)$. As $\Delta_{E_{k}} \neq \rho \cap \theta \subsetneq \theta \neq \nabla_{E_{k}}$, the sufficiency part yields $\operatorname{Pol}(\theta) \cap \operatorname{Pol}(\rho \cap \theta)$ is maximal in $\operatorname{Pol}(\theta)$. 
Lemma 4.7. Let $\rho$ and $\theta$ be two nontrivial equivalence relations which are incomparable.

If $\rho \cap \theta=\Delta_{E_{k}}$ and $\rho \circ \theta \neq \nabla_{E_{k}}$, then for $\sigma=\rho \circ \theta \cap \theta \circ \rho$, we have $\operatorname{Pol}(\theta) \cap \operatorname{Pol}(\rho) \subsetneq \operatorname{Pol}(\theta) \cap \operatorname{Pol}(\sigma) \subsetneq \operatorname{Pol}(\theta)$.

Proof. By assumptions we have $\sigma \neq \nabla_{E_{k}}$, hence $\operatorname{Pol}(\theta) \cap \operatorname{Pol}(\sigma) \subsetneq$ $\operatorname{Pol}(\theta)$. From the definition of $\sigma$, we get $\operatorname{Pol}(\theta) \cap \operatorname{Pol}(\rho) \subseteq \operatorname{Pol}(\theta) \cap \operatorname{Pol}(\sigma)$. Let us prove that $\operatorname{Pol}(\theta) \cap \operatorname{Pol}(\rho) \subsetneq \operatorname{Pol}(\theta) \cap \operatorname{Pol}(\sigma)$; choose $a \theta b$ with $a \neq b$ and $u \rho v$ with $u \neq v$ and define the following unary operation $g$ on $E_{k}$ by:

$$
g(x)= \begin{cases}a & \text { if } x=u, \\ b & \text { otherwise }\end{cases}
$$

As $(a, b) \in \theta$, we have $g \in \operatorname{Pol}(\theta)$ and $g \in \operatorname{Pol}(\sigma) ;(\theta \subseteq \sigma)$. Since $(g(u), g(v))=(a, b),(a, b) \in \theta \backslash \Delta_{E_{k}}$ and $\rho \cap \theta=\Delta_{E_{k}}, g \notin \operatorname{Pol}(\rho)$. Hence $g \notin \operatorname{Pol}(\theta) \cap \operatorname{Pol}(\rho)$ while $g \in \operatorname{Pol}(\theta) \cap \operatorname{Pol}(\sigma)$.

The two previous lemmas proved that if $\operatorname{Pol}(\theta) \cap \operatorname{Pol}(\rho)$ is a submaximal clone of $\operatorname{Pol}(\theta)$ and $\rho$ and $\theta$ are incomparable, then $\rho \cap \theta=\Delta_{E_{k}}$ and $\rho \circ \theta=\nabla_{E_{k}}$, so the condition (b) holds.

Proof (of Theorem 4.1). It follows from the previous lemmas.

We end this section with the following theorem due to Lau and Rosenberg, that deals with prime permutation relations.

Theorem 4.8 ([4]). If $\theta$ is a nontrivial equivalence relation and $\rho$ is a graph of prime permutation $\pi$, on a finite set $E_{k}$, then $\operatorname{Pol}(\theta) \cap \operatorname{Pol}(\rho)$ is submaximal in $\operatorname{Pol}(\theta)$ if and only if $\theta$ and $\rho$ satisfy one of the following statements: 
(a) $\rho \subsetneq \theta$

(b) The image of an equivalence class of $\theta$ is include in another class of $\theta$ surjectively.

\section{Central Relations and $h$-Regular Relations}

In this section, $\theta$ is a nontrivial equivalence relation on $E_{k}$, whose equivalence classes are: $C_{0}, C_{1}, \ldots, C_{t-1}$. Our aim is to characterize the central relations or $h$-regular relations $\rho$ such that $\operatorname{Pol}(\rho) \cap \operatorname{Pol}(\theta)$ is maximal in $\operatorname{Pol}(\theta)$.

Firstly, we give some definitions to be used. Let $\rho$ be an $h$-ary relation $(h>1)$ on $E_{k}$. For $i \in\{0 ; 1 ; 2 ; \cdots ; h-1\}$, we define the relation $\rho_{i, \theta}$ by

$$
\rho_{0, \theta}=\left\{\left(a_{1}, \ldots, a_{h}\right) \in E_{k}^{h} / \exists u_{i} \in\left[a_{i}\right]_{\theta}, i \in\{1 ; \cdots ; h\} \text { with }\left(u_{1}, u_{2}, \ldots, u_{h}\right) \in \rho\right\}
$$

and for $i \geq 1$,

$$
\begin{array}{r}
\rho_{i, \theta}=\left\{\left(a_{1}, \ldots, a_{h}\right) \in E_{k}^{h} / \exists u_{j} \in\left[a_{j}\right]_{\theta}, j \in\{i+1 ; \cdots ; h\}\right. \\
\text { with } \left.\left(a_{1}, a_{2}, \ldots, a_{i}, u_{i+1}, \ldots, u_{h}\right) \in \rho\right\} .
\end{array}
$$

For $\sigma \in \mathcal{S}_{h}$ and $\gamma$ an $h$-ary relation, we set

$$
\gamma_{\sigma}=\left\{\left(a_{\sigma(1)}, \ldots, a_{\sigma(h)}\right) /\left(a_{1}, \ldots, a_{h}\right) \in \gamma\right\}
$$

For $J=\left\{j_{i} ; \ldots ; j_{n}\right\} \subseteq\{1 ; \ldots ; h\}$ with $j_{1}<\ldots<j_{n}$, we define the $h$-ary relation $\rho_{J}$ or $\rho_{j_{1} \ldots j_{n}}$ on $E_{k}$ as follow:

$$
\rho_{J}=\left\{\left(a_{1}, a_{2}, \ldots, a_{h}\right) \mid \exists u_{i, J} \in\left[a_{i}\right]_{\theta}, i \in\{1 ; \cdots ; h\} \backslash J=\left\{i_{1}, \ldots, i_{h-n}\right\}\right.
$$

such that $\left.\left(a_{j_{1}}, \ldots, a_{j_{n}}, u_{i_{1}, J}, \ldots, u_{i_{h-n}, J}\right) \in \rho\right\}$.

The next remark gives some properties of those relations. 


\section{Remark 5.1.}

(1) For $i \in\{0 ; 1 ; 2 ; \cdots ; h-1\}, \rho \subseteq \rho_{i, \theta}$ and $\operatorname{Pol}(\rho) \cap \operatorname{Pol}(\theta) \subseteq \operatorname{Pol}\left(\rho_{i, \theta}\right)$.

(2) $\rho_{0, \theta}$ is totally symmetric.

(3) For $\sigma \in \mathcal{S}_{h}, \operatorname{Pol}(\rho)=\operatorname{Pol}\left(\rho_{\sigma}\right)$.

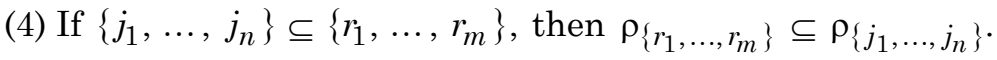

(5) For $J=\{1 ; \cdots ; n\}$ we have $\rho_{J}=\rho_{n, \theta}$.

(6) For all $1 \leq j_{1}<\ldots<j_{n} \leq h$, there exists a permutation $\sigma \in \mathcal{S}_{h}$ such that $\rho_{j_{1} \ldots j_{n}}=\left(\rho_{n, \theta}\right)_{\sigma}$.

Definition 5.2. Let $\rho$ be an $h$-ary relation and $\theta$ be a nontrivial equivalence relation on $E_{k}$ with $t$ classes.

(1) There is a transversal $T$ for the $\theta$-classes means that there exist $u_{1}, \ldots, u_{t} \in E_{k}$ such that $\left(u_{i}, u_{j}\right) \notin \theta$ for all $1 \leq i<j \leq t,\left(u_{i_{1}}, u_{i_{2}}, \ldots\right.$, $\left.u_{i_{h}}\right) \in \rho$ for all $1 \leq i_{1}, \ldots, i_{h} \leq t$ and $T=\left\{u_{1}, \ldots, u_{t}\right\}$.

(2) There is a transversal $T$ of order $l(1 \leq l \leq h-1)$ for the $\theta$-classes means that there exist $u_{1}, \ldots, u_{t} \in E_{k}$ such that $\left(u_{i}, u_{j}\right) \notin \theta$ for all $1 \leq i<j \leq t,\left(a_{1}, a_{2}, \ldots, a_{l}, v_{l+1}, v_{l+2}, \ldots, v_{h}\right) \in \rho$, for all $a_{1}, a_{2}, \ldots, a_{l}$ $\in E_{k}$ and $v_{l+1}, v_{l+2}, \ldots, v_{h} \in\left\{u_{1} ; \ldots ; u_{t}\right\}$; and $T=\left\{u_{1}, \ldots, u_{t}\right\}$.

Definition 5.3. A transversal of order 0 for the $\theta$-classes means a transversal for the $\theta$-classes.

Definition 5.4. Let $\rho$ be an $h$-ary relation and $\theta$ be a nontrivial equivalence relation on $E_{k}$ with $t$ classes.

(1) $\rho$ is $\theta$-closed means that $\rho=\rho_{0, \theta}$.

(2) $\rho$ is weakly $\theta$-closed of order $l(1 \leq l \leq h-1)$ means that there is a transversal $T=\left\{u_{1} ; \cdots ; u_{t}\right\}$ of order $l-1$ for the $\theta$-classes and $\rho=\bigcap_{\sigma \in \mathcal{S}_{h}}\left(\rho_{l, \theta}\right)_{\sigma}$. 
Secondly, we characterize some particular relations. We consider the surjective map

$$
\begin{aligned}
\varphi: E_{k} & \rightarrow E_{t} \\
x & \mapsto \varphi(x)=i \text { if } x \in C_{i} .
\end{aligned}
$$

For an $n$-ary relation $\alpha$ on $E_{t}$, we set

$$
\varphi^{-1}(\alpha)=\left\{\left(a_{1}, \ldots, a_{n}\right) \in E_{k}^{n}:\left(\varphi\left(a_{1}\right), \varphi\left(a_{2}\right), \ldots, \varphi\left(a_{n}\right)\right) \in \alpha\right\}
$$

for an $n$-ary relation $\beta$ on $E_{k}$, we set

$$
\varphi(\beta)=\left\{\left(\varphi\left(a_{1}\right), \varphi\left(a_{2}\right), \ldots, \varphi\left(a_{n}\right)\right):\left(a_{1}, \ldots, a_{n}\right) \in \beta\right\} .
$$

Remark 5.5. With the previous considerations and for a central relation $\rho$, we have:

- $\rho$ is $\theta$-closed if and only if there exists an $h$-ary central relation $\gamma$ on $E_{t}$ such that $\rho=\varphi^{-1}(\gamma)$.

- If $\rho$ is weakly $\theta$-closed of order $l(1 \leq l \leq h-1)$, then $\operatorname{Pol}\left(\left(\rho_{l, \theta}\right)_{\sigma_{1}}\right.$ $\left.\cap \cdots \cap\left(\rho_{l, \theta}\right)_{\sigma_{n}}\right) \subseteq \operatorname{Pol}(\rho)$ for $\left\{\sigma_{1} ; \cdots ; \sigma_{n}\right\} \subseteq \mathcal{S}_{h}$.

Remark 5.6. Let $\rho$ be a binary relation and $\theta$ be a nontrivial equivalence relation on $E_{k}$ with $t$ classes.

(1) $\rho$ is $\theta$-closed if and only if $\rho=\theta \circ \rho \circ \theta$,

(2) $\rho$ is weakly $\theta$-closed of order 1 (or simply weakly $\theta$-closed) if and only if $\rho=(\theta \circ \rho) \cap(\rho \circ \theta)$, and there is a transversal $T$ for the $\theta$-classes.

Thirdly, we characterize the central relations $\rho$ generating the submaximality.

5.1. Central relations. We recall that for $k=2$, we have the result in the Post's description. If $k \geq 3$ and $h \in\{1 ; 2\}$ the following results give the characterization of existing submaximal classes. 
Lemma 5.7 ([7]). If $h=1$, then $\operatorname{Pol}(\rho) \cap \operatorname{Pol}(\theta)$ is maximal in $\operatorname{Pol}(\theta)$ if and only if the following condition is valid:

$$
\left(\exists I \subset\{0 ; \cdots ; t-1\}: \rho=\bigcup_{i \in I} C_{i}\right) \vee \forall j \in\{0 ; \cdots ; t-1\}, \rho \cap C_{j} \neq \emptyset .
$$

Theorem 5.8 ([18]). If $h=2$, then $\operatorname{Pol}(\rho) \cap \operatorname{Pol}(\theta)$ is maximal in $\operatorname{Pol}(\theta)$ if and only if one of the following conditions is valid:

(i) $\theta \subseteq \rho$ and every $\theta$-class contains a central element of $\rho$;

(ii) $\rho$ is $\theta$-closed;

(iii) $\rho$ is weakly $\theta$-closed of order 1 .

In the remaining of this subsection we suppose that $h \geq 3$ and $k \geq 3$.

For a nontrivial equivalence relation $\theta$, we define the $h$-ary relation $\eta$ by

$$
\eta=\left\{\left(u_{1}, u_{2}, \ldots, u_{h}\right) \in E_{k}^{h} / u_{1} \theta u_{2}\right\} .
$$

Here we state the main theorem of this subsection:

Theorem 5.9. Let $k \geq 3$ and let $\theta$ be a nontrivial equivalence relation on $E_{k}$ with $t$ equivalence classes. For an h-ary central relation $\rho$ on $E_{k}$, the clone $\operatorname{Pol}(\rho) \cap \operatorname{Pol}(\theta)$ is a submaximal clone of $\operatorname{Pol}(\theta)$ if and only if $h \leq t$ and $\rho$ satisfies one of the following three conditions:

I. $\eta \subseteq \rho$ and every $\theta$-class contains a central element of $\rho$.

II. $\rho$ is $\theta$-closed.

III. $\rho$ is weakly $\theta$-closed of order $l$ and $\eta \subseteq \rho$.

The proof of Theorem 5.9 will follow from the results obtained below. It will be given at the end of this subsection. 
Definition 5.10. Let $l \in\{$ I; II; III $\}$, $\rho$ is of type $l$ if $\rho$ satisfies the condition $l$ of Theorem 5.9.

The following examples clarify the type of relations defined above.

Example 5.11. Let $k \geq 3$ be an integer and $0 \leq i<j<r<n \leq k-1$, we denote by $A_{i, j, r}$ and $A_{i, j, r, n}$ the sets

$$
A_{i, j, r}:=\left\{(\sigma(i), \sigma(j), \sigma(r)) ; \sigma \in \mathcal{S}_{\{i ; j ; r\}}\right\}
$$

and

$$
A_{i, j, r, n}:=\left\{(\sigma(i), \sigma(j), \sigma(r), \sigma(n)) ; \sigma \in \mathcal{S}_{\{i ; j ; r ; n\}}\right\}
$$

We consider the following equivalence relations $\theta_{i}$ defined by their equivalence classes denoted by $C_{m}^{i}$ :

$\theta_{1}$ is defined on $E_{6}$ by $C_{0}^{1}=\{0 ; 1\}, C_{1}^{1}=\{2 ; 3\}, C_{2}^{1}=\{4 ; 5\}$;

$\theta_{2}$ is defined on $E_{5}$ by $C_{0}^{2}=\{0 ; 1\}, C_{1}^{2}=\{2\}, C_{2}^{2}=\{3\}, C_{3}^{2}=\{4\}$;

$\theta_{3}$ is defined on $E_{4}$ by $C_{0}^{3}=\{0 ; 1\}, C_{1}^{3}=\{2\}, C_{2}^{3}=\{3\}$;

$\theta_{4}$ is defined on $E_{8}$ by $C_{0}^{4}=\{0 ; 1 ; 2\}, C_{1}^{4}=\{3 ; 4 ; 5\}, C_{2}^{4}=\{6 ; 7\}$;

$\theta_{5}$ is defined on $E_{8}$ by $C_{0}^{5}=\{0 ; 1 ; 2\}, C_{1}^{5}=\{3 ; 4\}, C_{2}^{5}=\{5 ; 6\}$, $C_{3}^{5}=\{7\}$

and the relations

$$
\begin{gathered}
\Upsilon_{1}=E_{6}^{3} \backslash A_{1,2,5}, \Upsilon_{2}=E_{5}^{3} \backslash A_{2,3,4}, \Upsilon_{3}=E_{4}^{3} \backslash A_{1,2,3}, \\
\Upsilon_{4}=E_{8}^{3} \backslash\left(A_{1,4,6} \cup A_{1,4,7} \cup A_{1,5,7} \cup A_{1,5,6} \cup A_{2,3,7} \cup A_{2,4,6} \cup A_{2,4,7}\right. \\
\left.\cup A_{2,5,6} \cup A_{2,5,7}\right), \\
\Upsilon_{5}=E_{8}^{4} \backslash\left(A_{1,4,6,7} \cup A_{2,4,6,7}\right) .
\end{gathered}
$$


It is easy to see that: $\Upsilon_{1}$ is a central relation of type I with $\theta_{1} ; \Upsilon_{2}$ is a central relation of type II but not of type I with $\theta_{2}$; with $\theta_{3}, \Upsilon_{3}$ is a central relation whose center is $\{0\}$ but it is neither of type, I, II or III.

$\Upsilon_{4}$ is weakly $\theta_{4}$-closed of order 2 with a transversal of order 1 , $T_{1}=\{0 ; 3 ; 6\}$;

$\Upsilon_{5}$ is weakly $\theta_{5}$-closed of order 3 with a transversal of order 2 , $T_{2}=\{0 ; 3 ; 5 ; 7\}$.

Definition 5.12. Let $\theta$ be an equivalence relation on $E_{k}$. An $h$-ary relation $\tau$ on $E_{k}$ is said to be diagonal through $\theta$ if there exists an equivalence relation $\varepsilon_{1}$ on $\{1 ; 2 ; \ldots ; h\}$ with equivalence classes $A_{1}, A_{2}$, $\ldots, A_{l}$ and an equivalence relation $\varepsilon_{2}$ on $\left\{\min \left(A_{m}\right) ; 1 \leq m \leq l\right\}$ such that

$\tau=\left\{\left(a_{1}, a_{2}, \ldots, a_{h}\right) \in E_{k}^{h} /\left((i, j) \in \varepsilon_{1} \Rightarrow a_{i}=a_{j}\right)\right.$ and $\left.\left((i, j) \in \varepsilon_{2} \Rightarrow a_{i} \theta a_{j}\right)\right\}$.

Given two equivalence relations $\theta_{1}$ and $\theta_{2}$ satisfying Definition 5.12, we denote by $D_{\theta_{1} \theta_{2}}$ the corresponding diagonal relation through $\theta$.

\subsubsection{Proof of the necessity criterion in Theorem 5.9}

Proposition 5.13. If $k \geq 3, \theta$ is a nontrivial equivalence relation on $E_{k}$, and $\rho$ is an h-ary central relation on $E_{k}$ such that one of conditions I-III is satisfied, then the clone $\operatorname{Pol}(\rho) \cap \operatorname{Pol}(\theta)$ is a submaximal clone of $\operatorname{Pol}(\theta)$.

Before the proof of Proposition 5.13, we give some results characterizing relations containing $\operatorname{Pol}(\rho) \cap \operatorname{Pol}(\theta)$ and we show that $\operatorname{Pol}(\rho) \cap \operatorname{Pol}(\theta)$ contains an $h$-near-unanimity operation.

For the proof of this proposition we choose a fixed central element $c$ of $\rho$ and denote by $C_{\rho}$ the set of all central elements of $\rho$. If $\rho$ is of type I, choose a central element $c_{B}$ from the $\theta$-class $B$ which can be min 
$\left(B \cap C_{\rho}\right.$ ), and if $\rho$ is of type III and of order $l$, choose a transversal $T$ of order $l-1$ of the $\theta$-classes and denote by $T_{B}$ the element of $T$ representing the $\theta$-class $B$.

We begin with the following lemma characterizing the diagonal relations through $\theta$.

Lemma 5.14. For an equivalence relation $\theta$ on $E_{k}$ and a diagonal relation $\tau$ through $\theta$, with arity $h$ on $E_{k}$, we have $\operatorname{Pol}(\tau)=\operatorname{Pol}(\theta)$ or $\operatorname{Pol}(\tau)=\mathcal{O}\left(E_{k}\right)$.

Proof. Let $\theta$ be an equivalence relation on $E_{k}$ and $\tau=D_{\varepsilon_{1} \varepsilon_{2}}$ be a diagonal relation through $\theta$. Let $T=\left\{\min A_{m} ; 1 \leq m \leq l\right\}$, where $A_{m}, 1 \leq m \leq l$ are as in Definition 5.12. We distinguish two cases: (a) $\varepsilon_{2} \neq \Delta_{T}$ and (b) $\varepsilon_{2}=\Delta_{T}$.

(a) Assume that $\varepsilon_{2} \neq \Delta_{T}$.

There exist $u, v \in T$ with $u<v$ such that for all $\left(a_{1}, a_{2}, \ldots, a_{h}\right) \in \tau$, we have $\left(a_{u}, a_{v}\right) \in \theta$. Using the definition of $\tau$, we have $\operatorname{Pol}(\theta) \subseteq \operatorname{Pol}(\tau)$. By setting

$$
p r_{u v}(\tau):=\left\{\left(e_{u}^{h}(\mathbf{a}), e_{v}^{h}(\mathbf{a})\right) ; \mathbf{a}=\left(a_{1}, a_{2}, \ldots, a_{h}\right) \in \tau\right\},
$$

it follows that $p r_{u v}(\tau)=\theta$, therefore $\operatorname{Pol}(\tau) \subseteq \operatorname{Pol}(\theta)$, and it appears that $\operatorname{Pol}(\tau)=\operatorname{Pol}(\theta)$

(b) Assume that $\varepsilon_{2}=\Delta_{T}$.

It is clear that

$$
\tau=D_{\varepsilon_{1} \Delta_{T}}=\left\{\left(a_{1}, a_{2}, \ldots, a_{h}\right) \in E_{k}^{h} / i \varepsilon_{1} j \Rightarrow a_{i}=a_{j}\right\} .
$$

Hence $\operatorname{Pol}(\tau)=\mathcal{O}\left(E_{k}\right)$. 
Lemma 5.15. Under the assumptions of Proposition 5.13, we have:

(a) $\eta \subseteq \rho ;$

(b) If $\rho$ is of type I or II, then an h-ary relation $\tau$ on $E_{k}$ is preserved by every operation in $\operatorname{Pol}(\rho) \cap \operatorname{Pol}(\theta)$ if and only if $\tau$ is either the empty relation, a diagonal relation through $\theta$, or the relation $\rho$;

(c) If $\rho$ is of type III and of order $l$, then an h-ary relation $\tau$ on $E_{k}$ is preserved by every operation in $\operatorname{Pol}(\rho) \cap \operatorname{Pol}(\theta)$ if and only if $\tau$ is either the empty relation, a diagonal relation through $\theta$, or an intersection of relations of the form $\left(\rho_{l, \theta}\right)_{\sigma}$ with $\sigma \in \mathcal{S}_{h}$.

Proof. (a) The proof of $\eta \subseteq \rho$ is straightforward.

(b) Assume $\tau$ is either the empty relation, or a diagonal relation through $\theta$. Then $\operatorname{Pol}(\tau) \in\left\{\operatorname{Pol}(\theta) ; \mathcal{O}\left(E_{k}\right)\right\}$ (see Lemma 5.14); hence $\operatorname{Pol}(\rho) \cap \operatorname{Pol}(\theta) \subseteq \operatorname{Pol}(\tau)$. Assume $\tau$ is of the form $\left(\rho_{l, \theta}\right)_{\sigma}$. Then it is easy to see that $\operatorname{Pol}(\rho) \cap \operatorname{Pol}(\theta) \subseteq \operatorname{Pol}\left(\left(\rho_{l, \theta}\right)_{\sigma}\right)$. Hence, if $\tau$ is an intersection of relations of the form $\left(\rho_{l, \theta}\right)_{\sigma}$, then $\operatorname{Pol}(\rho) \cap \operatorname{Pol}(\theta) \subseteq \operatorname{Pol}(\tau)$.

Conversely, assume $\tau$ is preserved by all operations in $\operatorname{Pol}(\rho) \cap \operatorname{Pol}(\theta)$.

Let us suppose that $\tau$ is not the empty relation. We have to prove that $\tau$ is either a diagonal relation through $\theta$, or an intersection of relations of the form $\left(\rho_{l, \theta}\right)_{\sigma}$ with $l$ the order of $\rho$ and $\sigma \in \mathcal{S}_{h}$.

For this purpose, we define two equivalence relations. The first one denoted by $\epsilon_{1}$ is defined on $\{1 ; 2 ; \ldots ; h\}$ by:

$$
i \epsilon_{1} j \text { iff } \forall\left(a_{1}, a_{2}, \ldots, a_{h}\right) \in \tau, a_{i}=a_{j} .
$$

$\epsilon_{1}$ is an equivalence relation with classes $A_{0}, A_{1}, \ldots, A_{m}$. The second one, denoted by $\epsilon_{2}$ is defined on $T=\left\{\min \left(A_{i}\right) ; 0 \leq i \leq m\right\}$ by: 


$$
i \epsilon_{2} j \text { iff } \forall\left(a_{1}, a_{2}, \ldots, a_{h}\right) \in \tau,\left(a_{i}, a_{j}\right) \in \theta .
$$

It follows that $D_{\epsilon_{1} \epsilon_{2}}$ is a diagonal relation through $\theta$. In order to complete the proof of this lemma, we distinguish two cases: $\left(\epsilon_{1} \neq \Delta_{\{1 ; 2 ; \ldots ; h\}}\right.$ or $\left.\epsilon_{2} \neq \Delta_{T}\right)$ and $\left(\epsilon_{1}=\Delta_{\{1 ; 2 ; \ldots ; h\}}\right.$ and $\left.\epsilon_{2}=\Delta_{T}\right)$.

(i) We suppose that $\epsilon_{1} \neq \Delta_{\{1 ; 2 ; \ldots ; h\}}$ or $\epsilon_{2} \neq \Delta_{T}$. We prove that $D_{\epsilon_{1} \epsilon_{2}}=\tau$. It suffices to show that $D_{\epsilon_{1} \epsilon_{2}} \subseteq \tau ; \tau \subseteq D_{\epsilon_{1} \epsilon_{2}}$ by definitions of $\epsilon_{1}$ and $\epsilon_{2}$. We need only consider three subcases: $\left(\epsilon_{1}=\nabla_{\{1 ; 2 ; \ldots ; h\}}\right)$, $\left(\epsilon_{1} \neq \nabla_{\{1 ; 2 ; \ldots ; h\}}\right.$ and $\left.\epsilon_{2}=\nabla_{T}\right)$, and $\left(\epsilon_{1} \neq \nabla_{\{1 ; 2 ; \ldots ; h\}}\right.$ and $\left.\epsilon_{2} \neq \nabla_{T}\right)$.

(a) If $\epsilon_{1}=\nabla_{\{1 ; 2 ; \ldots ; h\}}$, then $D_{\epsilon_{1} \epsilon_{2}}=\left\{(x, \ldots, x) ; x \in E_{k}\right\}$. Since $\tau \subseteq D_{\epsilon_{1} \epsilon_{2}}$ and $\tau$ is not the empty relation, for each $b \in E_{k}$ the constant function of value $b$ preserves $\theta$ and $\rho$; hence $(b, \ldots, b) \in \tau$ and $\tau=D_{\epsilon_{1} \epsilon_{2}}$.

(b) If $\epsilon_{1} \neq \nabla_{\{1 ; \ldots ; h\}}$ and $\epsilon_{2}=\nabla_{T}$, then there exists $(i, j) \in \nabla_{\{1 ; \ldots ; h\}}$ such that $(i, j) \notin \epsilon_{1}$. For all $1 \leq i, j \leq h$ such that $(i, j) \notin \epsilon_{1}$, we choose $\mathbf{a}^{i j}=\left(a_{1}^{i j}, \ldots, a_{h}^{i j}\right) \in \tau$ such that $a_{i}^{i j} \neq a_{j}^{i j}$ and we set $B=\left\{\mathbf{a}^{i j} ;(i, j) \notin \epsilon_{1}\right\}$. Let $q=|B|$; to simplify our notation, we suppose that $B=\left\{\mathbf{b}^{1} ; \mathbf{b}^{2}\right.$; $\left.\ldots ; \mathbf{b}^{q}\right\}$ and we define the sequence $\left(\mathbf{x}_{i}\right)_{1 \leq i \leq h}$ by $\mathbf{x}_{i}=\left(b_{i}^{1} ; b_{i}^{2} ; \ldots ; b_{i}^{q}\right)$. It is easy to see that: $(i, j) \notin \epsilon_{1} \Rightarrow \mathbf{x}_{i} \neq \mathbf{x}_{j}$. Let $\left(u_{1}, \ldots, u_{h}\right) \in D_{\epsilon_{1} \epsilon_{2}}$, we consider the $q$-ary operation defined on $E_{k}$ by:

$$
f(\mathbf{y})= \begin{cases}u_{l} & \text { if } \mathbf{y}=\mathbf{x}_{l} \in\left\{\mathbf{x}_{1} ; \mathbf{x}_{2} ; \ldots ; \mathbf{x}_{h}\right\}, \\ u_{1} & \text { otherwise }\end{cases}
$$

Since $\left\{u_{1}, u_{2}, \ldots, u_{h}\right\}^{2} \subseteq \theta$ and $\eta \subseteq \rho, f \in \operatorname{Pol}(\theta) \cap \operatorname{Pol}(\rho)$. Therefore $f \in \operatorname{Pol}(\tau)$ and $\left(u_{1}, \ldots, u_{h}\right) \in \tau$. 
(c) If $\epsilon_{1} \neq \nabla_{\{1 ; 2 ; \ldots ; h\}}$ and $\epsilon_{2} \neq \nabla_{T}$, then there exists $(i, j) \in \nabla_{T}$ such that $(i, j) \notin \epsilon_{2}$. For all $1 \leq i, j \leq h$ such that $(i, j) \notin \epsilon_{2}$ we choose $\mathbf{a}^{i j}=\left(a_{1}^{i j}, a_{2}^{i j}, \ldots, a_{h}^{i j}\right) \in \tau$ such that $\left(a_{i}^{i j}, a_{j}^{i j}\right) \notin \theta$ and we consider the set $B=\left\{\mathbf{a}^{i j},(i, j) \notin \epsilon_{2}\right\}$. We set $q=|B|$. To simplify our notation we set $B=\left\{\mathbf{b}^{1} ; \mathbf{b}^{2} ; \ldots ; \mathbf{b}^{q}\right\}$ which allows us to define $\mathbf{x}_{1}=\left(b_{1}^{1}, b_{1}^{2}, \ldots, b_{1}^{q}\right), \mathbf{x}_{2}$ $=\left(b_{2}^{1}, b_{2}^{2}, \ldots, b_{2}^{q}\right), \ldots, \mathbf{x}_{s}=\left(b_{s}^{1}, b_{s}^{2}, \ldots, b_{s}^{q}\right)$. We remark that $(i, j) \notin \epsilon_{2}$ $\Rightarrow\left(\mathbf{x}_{i}, \mathbf{x}_{j}\right) \notin \theta$.

Let $\left(u_{1}, u_{2}, \ldots, u_{h}\right) \in D_{\epsilon_{1} \epsilon_{2}}$ and consider the $q$-ary operation defined on $E_{k}$ by:

$$
f(\mathbf{y})= \begin{cases}u_{l} & \text { if } \mathbf{y}=\mathbf{x}_{l}, \\ u_{\sigma(\mathbf{y})} & \text { if } \mathbf{y} \notin\left\{\mathbf{x}_{1} ; \mathbf{x}_{2} ; \ldots ; \mathbf{x}_{h}\right\}, \exists l \in\{1,2, \ldots, h\} / \mathbf{y} \theta \mathbf{x}_{l} \text { and } \\ & \sigma(\mathbf{y})=\min \left\{t / \mathbf{y} \theta \mathbf{x}_{t}\right\} \\ c & \text { otherwise, }\end{cases}
$$

where $c$ is a central element. Since $\rho$ is totally reflexive and $D_{\epsilon_{1} \epsilon_{2}} \subseteq \rho$, it follows that $f \in \operatorname{Pol}(\rho)$. By the definition of $f$, we have $f \in \operatorname{Pol}(\theta)$, thus $f \in \operatorname{Pol}(\tau)$. Since $\mathbf{b}^{1}, \ldots, \mathbf{b}^{q} \in \tau$, it follows that $f\left(\mathbf{b}^{1}, \mathbf{b}^{2}, \ldots, \mathbf{b}^{q}\right)=$ $\left(f\left(\mathbf{x}_{1}\right), f\left(\mathbf{x}_{2}\right), \ldots, f\left(\mathbf{x}_{h}\right)\right)=\left(u_{1}, u_{2}, \ldots, u_{h}\right) \in \tau$, therefore $D_{\epsilon_{1} \epsilon_{2}} \subseteq \tau$.

(ii) We suppose that $\epsilon_{1}=\Delta_{\{1 ; 2 ; \ldots ; h\}}$ and $\epsilon_{2}=\Delta_{T}$. We show that $\tau$ is an intersection of relations of the form $\left(\rho_{l, \theta}\right)_{\sigma}$ or the relation $E_{k}^{h}$.

For all $1 \leq i, j \leq h$ such that $i \neq j$, choose $\mathbf{a}^{i j}=\left(a_{1}^{i j}, \ldots, a_{s}^{i j}\right) \in \tau$ such that $\left(a_{i}^{i j}, a_{j}^{i j}\right) \notin \theta$ and consider the set $B=\left\{\mathbf{a}^{i j},(i, j) \notin \epsilon_{2}\right\}$. Using similar notation as in part (c) of (i) and the same $q$-ary operation $f$ on $E_{k}$ for a given $\left(u_{1}, u_{2}, \ldots, u_{h}\right) \in \rho$, we have 


$$
f\left(\mathbf{b}^{1}, \mathbf{b}^{2}, \ldots, \mathbf{b}^{q}\right)=\left(f\left(\mathbf{x}_{1}\right), f\left(\mathbf{x}_{2}\right), \ldots, f\left(\mathbf{x}_{h}\right)\right)=\left(u_{1}, u_{2}, \ldots, u_{h}\right) \in \tau,
$$

and then $\rho \subseteq \tau$. By the definition of $\left(\rho_{l, \theta}\right)_{\sigma}$ with $l$ the order of $\rho$ and $\sigma \in \mathcal{S}_{h}$, we have: $\rho \subseteq\left(\rho_{l, \theta}\right)_{\sigma}$ for all $\sigma \in \mathcal{S}_{h}$. We distinguish once more two subcases: $\rho=\tau$ or $\rho \neq \tau$.

(a) If $\rho=\tau$, then it is finished.

(b) Otherwise $\rho \subsetneq \tau \subseteq E_{k}^{h}$.

If $\rho$ is of type I or II, then we show that $\tau=E_{k}^{h}$.

As $\rho \subsetneq \tau$ then $\tau \backslash \rho \neq \emptyset$. Let us consider $\left(a_{1}, a_{2}, \ldots, a_{h}\right) \in \tau \backslash \rho$. Let $\left(u_{1}, u_{2}, \ldots, u_{h}\right) \in E_{k}^{h}$; assume $\rho$ is of type I and consider the unary operation $f$ defined on $E_{k}$ by:

$$
f(x)= \begin{cases}u_{i} & \text { if } x=a_{i} \\ c_{\left[a_{i}\right]_{\theta}} & \text { if } x \theta a_{i} \text { and } x \neq a_{i} \\ c & \text { otherwise }\end{cases}
$$

where $c$ is a central element. $f \in \operatorname{Pol}(\rho)$ because $\eta \subseteq \rho$ and $\rho$ is totally symmetric. Moreover $f \in \operatorname{Pol}(\theta)$, then $f \in \operatorname{Pol}(\tau)$ and $\left(u_{1}, u_{2}, \ldots, u_{h}\right)=$ $\left(f\left(a_{1}\right), f\left(a_{2}\right), \ldots, f\left(a_{h}\right)\right) \in \tau$.

Assume $\rho$ is of type II and consider the unary operation $f$ defined on $E_{k}$ by:

$$
f(x)= \begin{cases}u_{i} & \text { if } x \theta a_{i} \\ c & \text { otherwise }\end{cases}
$$

where $c$ is a central element. $f \in \operatorname{Pol}(\rho)$ because $\rho$ is $\theta$-closed. Moreover $f \in \operatorname{Pol}(\theta)$, then $f \in \operatorname{Pol}(\tau)$ and $\left(u_{1}, u_{2}, \ldots, u_{h}\right)=\left(f\left(a_{1}\right)\right.$, 
$\left.f\left(a_{2}\right), \ldots, f\left(a_{h}\right)\right) \in \tau$. Thus $\tau=E_{k}^{h}=D_{\Delta\{1 ; \cdots ; h\}^{\Delta}\{1 ; \cdots ; h\}}$. Let's suppose that $\rho$ is of type III and of order $l$, i.e., $\rho$ is weakly $\theta$-closed of order $l$ and $\eta \subseteq \rho$.

Since $\tau \backslash \rho$ is not empty, there exists $\left(a_{1}, a_{2}, \ldots, a_{h}\right) \in(\tau \backslash \rho)$. Therefore $\left(a_{1}, a_{2}, \ldots, a_{h}\right) \notin \bigcap_{\sigma \in \mathcal{S}_{h}}\left(\rho_{l, \theta}\right)_{\sigma}$. We suppose that there is $\sigma^{\prime} \in \mathcal{S}_{h}$ such that $\left(a_{1}, a_{2}, \ldots, a_{h}\right) \in\left(\rho_{l, \theta}\right)_{\sigma^{\prime}}$. We consider the set $R_{1}:=\left\{\sigma^{\prime} \in \mathcal{S}_{h} /\left(a_{1}, a_{2}, \ldots, a_{h}\right) \in\left(\rho_{l, \theta}\right)_{\sigma^{\prime}}\right\}$ and define the relation $\varphi$ by

$$
\varphi=\bigcap_{\sigma^{\prime} \in R_{1}}\left(\rho_{l, \theta}\right)_{\sigma^{\prime}}
$$

We have $\rho \subsetneq \varphi$.

We show that $\varphi \subseteq \tau$. Let $\left(u_{1}, \ldots, u_{h}\right) \in \varphi$ and set

$$
\begin{array}{r}
D=\left\{\left(b_{1}, \ldots, b_{h}\right) \in E_{k}^{h} ; b_{i} \in\left\{u_{1}, \ldots, u_{h}\right\} \cup\left\{c, T_{\left[u_{1}\right]_{\theta}}, \ldots, T_{\left[u_{h}\right]_{\theta}}\right\}, 1 \leq i \leq h,\right. \\
\\
\text { and } \left.\left\{b_{1}, \ldots, b_{s}\right\} \neq\left\{u_{1}, \ldots, u_{h}\right\}\right\} .
\end{array}
$$

We define the unary operation $h$ on $E_{k}$ by:

$$
h(x)=\left\{\begin{array}{l}
u_{i} \text { if } x=a_{i}, \\
T_{\left[u_{i}\right]_{\theta}} \text { if } x \in\left[a_{i}\right]_{\theta} \backslash\left\{a_{i}\right\}, \\
c \text { otherwise. }
\end{array}\right.
$$

For $\left(y_{1}, \ldots, y_{h}\right) \in \rho$, we have $\left(h\left(y_{1}\right), \ldots, h\left(y_{h}\right)\right) \in D \cap \rho \subseteq \rho$. Therefore $h \in \operatorname{Pol}(\rho) \cap \operatorname{Pol}(\theta)$. Hence $h \in \operatorname{Pol}(\tau)$ and $\left(u_{1}, \ldots, u_{h}\right)=\left(h\left(a_{1}\right), \ldots\right.$, $\left.h\left(a_{h}\right)\right) \in \tau$.

If $\tau=\varphi$, then it is finished. Otherwise, we have $\varphi \subsetneq \tau$. 
There exists $\left(a_{1}, a_{2}, \ldots, a_{h}\right) \in \tau \backslash \varphi$. If there exists $s \in \mathcal{S}_{h}$ such that $\left(a_{1}, a_{2}, \ldots, a_{h}\right) \in\left(\rho_{l, \theta}\right)_{s}$, then we use the same argument to construct $\varphi^{\prime}$ such that $\varphi \subsetneq \varphi^{\prime}$ and $\varphi^{\prime} \subseteq \tau$. Therefore $\tau=\varphi^{\prime}$ or $\varphi^{\prime} \subsetneq \tau$. So we have the same conclusion as above. We continue this process until obtained a $h$-tuple $\left(a_{1}, a_{2}, \ldots, a_{h}\right) \in \tau$ such that, for each $\sigma \in \mathcal{S}_{h},\left(a_{1}\right.$, $\left.a_{2}, \ldots, a_{h}\right) \notin\left(\rho_{l, \theta}\right)_{\sigma}$. Let $\left(u_{1}, \ldots, u_{h}\right) \in E_{k}^{h}$, using the unary operation $h$ defined above and the fact that $\rho$ is weakly $\theta$-closed of order $l$ and there is a transversal $T$ of order $l-1$ for the $\theta$-classes, we show that $\left(u_{1}, \ldots, u_{h}\right) \in \tau$. Therefore $\tau=E_{k}^{h}$.

Lemma 5.16. Under the assumptions of Proposition 5.13, $\operatorname{Pol}(\theta) \cap \operatorname{Pol}(\rho)$ contains an h-near-unanimity operation.

Proof. If $\rho$ is of type I, let us consider the $(s+1)$-ary operation $m$ defined on $E_{k}$ by:

$$
m\left(x_{1}, \ldots, x_{h}, x_{h+1}\right)= \begin{cases}x_{i_{1}} & \text { if there exist } 1 \leq i_{1}<\ldots<i_{h} \leq h+1 \\ & \text { such that } x_{i_{1}}=x_{i_{2}}=\ldots=x_{i_{s}}, \\ c\left[x_{i_{1}}\right]_{\theta} & \text { if there exist } 1 \leq i_{1}<\ldots<i_{h} \leq h+1 \\ & \text { such that }\left[x_{i_{1}}\right]_{\theta}=\left[x_{i_{2}}\right]_{\theta}=\ldots=\left[x_{i_{s}}\right]_{\theta}, \\ c & \text { otherwise }\end{cases}
$$

if $\rho$ is of type II, let us consider the $(s+1)$-ary operation $m$ defined on $E_{k}$ by: 


$$
m\left(x_{1}, \ldots, x_{h}, x_{h+1}\right)= \begin{cases}x_{i_{1}} & \text { if there exist } 1 \leq i_{1}<\ldots<i_{h} \leq h+1 \\ & \text { such that } x_{i_{1}}=x_{i_{2}}=\ldots=x_{i_{s}}, \\ x_{i_{1}} & \text { if there exist } 1 \leq i_{1}<\ldots<i_{h} \leq h+1 \\ & \text { such that }\left[x_{i_{1}}\right]_{\theta}=\left[x_{i_{2}}\right]_{\theta}=\ldots=\left[x_{i_{h}}\right]_{\theta}, \\ c \quad & \text { otherwise; }\end{cases}
$$

if $\rho$ is of type III, let us consider the $(s+1)$-ary operation $m$ defined on $E_{k}$ by:

$$
m\left(x_{1}, \ldots, x_{h}, x_{h+1}\right)= \begin{cases}x_{i_{1}} & \text { if there exist } 1 \leq i_{1}<\ldots<i_{h} \leq h+1 \\ & \text { such that } x_{i_{1}}=x_{i_{2}}=\ldots=x_{i_{h}}, \\ T_{\left[x_{i_{1}}\right]_{\theta}} & \text { if there exist } 1 \leq i_{1}<\ldots<i_{h} \leq h+1 \\ & \text { such that }\left[x_{i_{1}}\right]_{\theta}=\left[x_{i_{2}}\right]_{\theta}=\ldots=\left[x_{i_{h}}\right]_{\theta}, \\ c & \text { otherwise; }\end{cases}
$$

where $c$ is a central element. By definition, $m$ is a near unanimity function of order $h+1$. We show that $m \in \operatorname{Pol}(\rho) \cap \operatorname{Pol}(\theta)$. To do this, we prove firstly that $m \in \operatorname{Pol}(\theta)$ and secondly that $m \in \operatorname{Pol}(\rho)$.

(a) To see that $m$ preserves $\theta$, assume that $\left(u_{i}, v_{i}\right) \in \theta$ for $1 \leq i \leq s+1$. Since

$$
\left|\left\{\left[u_{1}\right]_{\theta} ;\left[u_{2}\right]_{\theta} ; \ldots ;\left[u_{h+1}\right]_{\theta}\right\}\right|=\left|\left\{\left[v_{1}\right]_{\theta} ;\left[v_{2}\right]_{\theta} ; \ldots ;\left[v_{h+1}\right]_{\theta}\right\}\right|,
$$

if there exist $1 \leq i_{1}<i_{2}<\ldots<i_{h} \leq h+1$ such that $\left[u_{i_{1}}\right]_{\theta}=\left[u_{i_{2}}\right]_{\theta}=\ldots$ $=\left[u_{i_{h}}\right]_{\theta}$, then $m\left(u_{1}, u_{2}, \ldots, u_{h+1}\right) \in\left[u_{i_{1}}\right]_{\theta}=\left[v_{i_{1}}\right]_{\theta}$ because $\left[u_{i}\right]_{\theta}=\left[v_{i}\right]_{\theta}$ for $i=1,2, \ldots, h+1$.

Therefore, $\left(m\left(u_{1}, u_{2}, \ldots, u_{h+1}\right), m\left(v_{1}, v_{2}, \ldots, v_{h+1}\right)\right) \in\left[u_{i_{1}}\right]_{\theta}^{2} \subseteq \theta$. In the other case, we have $\left(m\left(u_{1}, u_{2}, \ldots, u_{h+1}\right), m\left(v_{1}, v_{2}, \ldots, u_{h+1}\right)\right)=(c, c) \in \theta$; then $m \in \operatorname{Pol}(\theta)$. 
(b) Let us prove now that $m$ preserves $\rho$.

Let $\left(u_{11}, u_{21}, \ldots, u_{h 1}\right),\left(u_{12}, u_{22}, \ldots, u_{h 2}\right), \ldots,\left(u_{1 h+1}, u_{2 h+1}, \ldots, u_{h h+1}\right) \in \rho$. If $\left\{m\left(u_{i 1}, u_{i 2}, \ldots, u_{i h+1}\right) ; 1 \leq i \leq h\right\}$ contains a central element of $\rho$, then

$$
\left(m\left(u_{11}, u_{12}, \ldots, u_{1 h+1}\right), \ldots, m\left(u_{h 1}, u_{h 2}, \ldots, u_{h h+1}\right)\right) \in \rho
$$

else we distinguish the three type of $\rho$.

Suppose $\rho$ be of type $\mathrm{I}$, there exist $1 \leq i_{r}^{1}<i_{r}^{2}<\ldots<i_{r}^{h} \leq h+1$, $1 \leq r \leq h$ such that $u_{r i_{r}^{1}}=u_{r i_{r}^{2}}=\ldots=u_{r i_{r}^{h}}$ for $r \in\{1 ; \cdots ; h\} ;$ as $\left\{i_{1}^{1} ; i_{1}^{2} ; \ldots\right.$; $\left.i_{1}^{h}\right\} \cap\left\{i_{2}^{1} ; i_{2}^{2} ; \ldots ; i_{2}^{h}\right\} \cap, \ldots, \cap\left\{i_{h}^{1} ; i_{h}^{2} ; \ldots ; i_{h}^{h}\right\} \neq \emptyset$, let us consider a fixed element $i \in\left\{i_{1}^{1} ; i_{1}^{2} ; \ldots ; i_{1}^{h}\right\} \cap\left\{i_{2}^{1} ; i_{2}^{2} ; \ldots ; i_{2}^{h}\right\} \cap, \ldots, \cap\left\{i_{h}^{1} ; i_{h}^{2} ; \ldots ; i_{h}^{h}\right\} . \quad \mathrm{We}$ have

$$
\left(m\left(u_{11}, u_{12}, \ldots, u_{1 h+1}\right), \ldots, m\left(u_{h 1}, u_{h 2}, \ldots, u_{h h+1}\right)\right)=\left(u_{1 i}, \ldots, u_{h i}\right) \in \rho .
$$

Therefore $m \in \operatorname{Pol}(\rho)$.

If $\rho$ is of type II there exist $1 \leq i_{r}^{1}<i_{r}^{2}<\ldots<i_{r}^{h} \leq h+1,1 \leq r \leq h$ such that $\left[u_{r i_{r}^{1}}\right]_{\theta}=\left[u_{r i_{r}^{2}}\right]_{\theta}=\ldots=\left[u_{r i_{r}^{h}}\right]_{\theta}$ for $r \in\{1 ; \cdots ; h\} ;$ as $\left\{i_{1}^{1} ; i_{1}^{2}\right.$; $\left.\ldots ; i_{1}^{h}\right\} \cap\left\{i_{2}^{1} ; i_{2}^{2} ; \ldots ; i_{2}^{h}\right\} \cap, \ldots, \cap\left\{i_{h}^{1} ; i_{h}^{2} ; \ldots ; i_{h}^{h}\right\} \neq \emptyset$, then with $i \in\left\{i_{1}^{1}\right.$; $\left.i_{1}^{2} ; \ldots ; i_{1}^{h}\right\} \cap\left\{i_{2}^{1} ; i_{2}^{2} ; \ldots ; i_{2}^{h}\right\} \cap \ldots, \cap\left\{i_{h}^{1} ; i_{h}^{2} ; \ldots ; i_{h}^{h}\right\}$ we have $\left(m\left(u_{11}, u_{12}, \ldots, u_{1 h+1}\right), \ldots, m\left(u_{h 1}, u_{h 2}, \ldots, u_{h h+1}\right)\right) \in\left[u_{1 i}\right]_{\theta} \times \ldots \times\left[u_{h i}\right]_{\theta} \subseteq \rho$ because $\rho$ is $\theta$-closed. Thus $m \in \operatorname{Pol}(\rho)$.

Finally, if $\rho$ is of type III, there exist $1 \leq i_{r}^{1}<i_{r}^{2}<\ldots<i_{r}^{h} \leq h+1,1$ $\leq r \leq h$ such that $\left[u_{r i_{r}^{1}}\right]_{\theta}=\left[u_{r i_{r}^{2}}\right]_{\theta}=\ldots=\left[u_{r i_{r}^{h}}\right]_{\theta}$ for $r \in\{1 ; \cdots ; h\}$; as

$$
\left\{i_{1}^{1} ; i_{1}^{2} ; \ldots ; i_{1}^{h}\right\} \cap\left\{i_{2}^{1} ; i_{2}^{2} ; \ldots ; i_{2}^{h}\right\} \cap \ldots, \cap\left\{i_{h}^{1} ; i_{h}^{2} ; \ldots ; i_{h}^{h}\right\} \neq \emptyset
$$


then with $i \in\left\{i_{1}^{1} ; i_{1}^{2} ; \ldots ; i_{1}^{h}\right\} \cap\left\{i_{2}^{1} ; i_{2}^{2} ; \ldots ; i_{2}^{h}\right\} \cap \ldots, \cap\left\{i_{h}^{1} ; i_{h}^{2} ; \ldots ; i_{h}^{h}\right\}$ we have (with $\left.Z:=\left(m\left(u_{11}, \ldots, u_{1 h+1}\right), \ldots, m\left(u_{h 1}, \ldots, u_{h h+1}\right)\right)\right)$

$$
Z \in\left\{u_{1 i}, \ldots, u_{h i}, T_{\left[u_{1 i}\right]_{\theta}}, \ldots, T_{\left[u_{h i}\right]_{\theta}}\right\}^{h} \subseteq \rho .
$$

Hence $m \in \operatorname{Pol}(\rho)$.

Proof (of Proposition 5.13). Let $f \in \operatorname{Pol}(\theta) \backslash(\operatorname{Pol}(\theta) \cap \operatorname{Pol}(\rho)$ ). We prove that $G=<\operatorname{Pol}(\theta) \cap \operatorname{Pol}(\rho) \cup\{f\}>$ is equal to $\operatorname{Pol}(\theta)$. From Lemma 5.16, it follows that $\operatorname{Pol}(\theta) \cap \operatorname{Pol}(\rho)$ contains an $h$-near-unanimity function $m$. According to Theorem 2.1 and the fact that $m \in G$, we have $G=\operatorname{PolInv} v^{(h)} G$. If $\tau \in \operatorname{Inv}^{(h)} G$, then $\operatorname{Pol}(\theta) \cap \operatorname{Pol}(\rho) \subseteq<\operatorname{Pol}(\theta) \cap \operatorname{Pol}(\rho)$ $\bigcup\{f\}>=G=\operatorname{Pol} I n v^{(h)} G \subseteq \operatorname{Pol}(\tau)$. By Lemma 5.15, if $\rho$ is of type I or II, then $\tau$ is either the empty relation, either a diagonal relation through $\theta$ or $\rho$; if $\rho$ is of type III and of order $l$, then $\tau$ is either the empty relation, either a diagonal relation through $\theta$ or an intersection of relations of the form $\left(\rho_{l, \theta}\right)_{\sigma}$ with $\sigma \in \mathcal{S}_{h}$. Since $f \notin \operatorname{Pol}(\rho)$, therefore $f$ can not preserve an intersection of relations of the form $\left(\rho_{l, \theta}\right)_{\sigma}$ with $\sigma \in \mathcal{S}_{h}$; therefore $\tau$ is the empty relation or a diagonal relation through $\theta$. In the light of Lemma 5.14, we have $\operatorname{Pol}(\theta) \subseteq G$.

Remark 5.17. Let us mention that if $\rho$ is of type I or II, then $\operatorname{Pol}(\theta)$ $\cap \operatorname{Pol}(\rho)$ is also maximal in $\operatorname{Pol}(\rho)$, whereas if $\rho$ is of type III, then $\operatorname{Pol}(\theta) \cap \operatorname{Pol}(\rho)$ is not maximal in $\operatorname{Pol}(\rho)$.

\subsubsection{Proof of the completeness criterion}

In this subsection, we show that the relations of type I, II and III are the only central relations $\rho$ such that $\operatorname{Pol}(\theta) \cap \operatorname{Pol}(\rho)$ is maximal in $\operatorname{Pol}(\theta)$. We assume that $\operatorname{Pol}(\theta) \cap \operatorname{Pol}(\rho)$ is maximal in $\operatorname{Pol}(\theta)$ and show that apart from these types, we don't have maximality. 
Proposition 5.18. If $\operatorname{Pol}(\rho) \cap \operatorname{Pol}(\theta)$ is maximal in $\operatorname{Pol}(\theta)$, then $\rho$ is either of type I, II, or III.

Before the proof of Proposition 5.18, we give some results on the properties of the relation $\rho$.

Lemma 5.19. If $\operatorname{Pol}(\rho) \cap \operatorname{Pol}(\theta)$ is maximal in $\operatorname{Pol}(\theta)$, then $\eta \subseteq \rho$.

Proof. By contraposition, suppose that $\eta \nsubseteq \rho$, therefore there exists

$$
\left(u_{1}, u_{2}, \ldots, u_{h}\right) \in \eta \quad \text { such that } \quad\left(u_{1}, u_{2}, \ldots, u_{h}\right) \notin \rho .
$$

We denote by $\eta^{1}$ the relation

$$
\eta^{1}=\left\{\left(u_{1}, u_{2}, \ldots, u_{h}\right) \in \rho / u_{1} \theta u_{2}\right\} .
$$

The following inclusions hold:

$$
\operatorname{Pol}(\rho) \cap \operatorname{Pol}(\theta) \subsetneq \operatorname{Pol}\left(\eta^{1}\right) \subsetneq \operatorname{Pol}(\theta) .
$$

(i) Let $f \in \operatorname{Pol}(\rho) \cap \operatorname{Pol}(\theta)$ with arity $n$, we prove that $f \in \operatorname{Pol}\left(\eta^{1}\right)$. Let $\left(u_{11}, u_{21}, \ldots, u_{h 1}\right), \ldots,\left(u_{1 n}, u_{2 n}, \ldots, u_{h n}\right) \in \eta^{1}$.

We have $f\left(u_{11}, \ldots, u_{1 n}\right) \theta f\left(u_{21}, \ldots, u_{2 n}\right)$ since $f \in \operatorname{Pol}(\theta)$ and

$$
\left(f\left(u_{11}, \ldots, u_{1 n}\right), f\left(u_{21}, \ldots, u_{2 n}\right), \ldots, f\left(u_{h 1}, \ldots, u_{h n}\right)\right) \in \rho
$$

since $f \in \operatorname{Pol}(\rho)$. Therefore $f \in \operatorname{Pol}\left(\eta^{1}\right)$.

Let $\left(u_{1}, u_{2}, \ldots, u_{h}\right) \in \eta \backslash \rho$ and $(a, b) \notin \theta$ fixed, we define the $h$-ary operation $f$ on $E_{k}$ by:

$$
f\left(x_{1}, x_{2}, \ldots, x_{h}\right)=\left\{\begin{array}{l}
u_{1} \text { if }\left(x_{1}, x_{2}, \ldots, x_{h}\right) \theta(a, a, \ldots, a), \\
u_{2} \text { if }\left(x_{1}, x_{2}, \ldots, x_{h}\right) \theta(a, b, a, \ldots, a), \\
\vdots \\
u_{h} \text { if }\left(x_{1}, x_{2}, \ldots, x_{h}\right) \theta(a, a, \ldots, a, b), \\
u_{1} \text { otherwise. }
\end{array}\right.
$$


With $\left(u_{11}, u_{21}, \ldots, u_{h 1}\right), \ldots,\left(u_{1 s}, u_{2 s}, \ldots, u_{s s}\right) \in \eta^{1}, \quad$ according $\quad$ to definition of $\eta^{1}$, we have $f\left(u_{11}, \ldots, u_{1 s}\right)=f\left(u_{21}, \ldots, u_{2 s}\right)$ since $f \in \operatorname{Pol}(\theta)$ and $\left(u_{11}, \ldots, u_{1 n}\right) \theta\left(u_{21}, \ldots, u_{2 n}\right)$. From the fact that $\rho$ is totally reflexive we have

$$
\left(f\left(u_{11}, \ldots, u_{1 n}\right), f\left(u_{21}, \ldots, u_{2 n}\right), \ldots, f\left(u_{h 1}, \ldots, u_{h n}\right)\right) \in \eta^{1} .
$$

Hence $f \in \operatorname{Pol}\left(\eta^{1}\right)$.

Furthermore, $\left(\begin{array}{llllll}a & a & a & \cdots & a & a \\ a & b & a & \cdots & a & a \\ \vdots & & & & & \\ a & a & a & \cdots & a & b\end{array}\right) \subseteq \rho$ and by the definition of $f$ we have

$\left(u_{1}, u_{2}, \ldots, u_{h}\right)=(f(a, a, a, \ldots, a), f(a, b, a, \ldots, a), \ldots, f(a, a, \ldots, a, b)) \notin \rho$.

Therefore, $f \notin \operatorname{Pol}(\rho)$ and $\operatorname{Pol}(\rho) \cap \operatorname{Pol}(\theta) \subsetneq \operatorname{Pol}\left(\eta^{1}\right)$.

(ii) From the equality $\operatorname{pr}_{12}\left(\eta^{1}\right)=\theta$, it follows that $\operatorname{Pol}\left(\eta^{1}\right) \subseteq \operatorname{Pol}(\theta)$. Let $\left(u_{1}, u_{2}, \ldots, u_{h}\right) \in \eta \backslash \rho$ be a fixed element and $c$ be a central element of $\rho$. It is obvious that $\left(u_{1}, u_{2}, \ldots, u_{h}\right) \notin \eta^{1}$. Let $a, b, c \in E_{k}$ such that $(a, b) \in \theta, a \neq b$ and $(a, c) \notin \theta$.

For $1 \leq i \leq h$, we define the $(h-1)$-tuple $\mathbf{W}_{i}=\left(w_{i 1}, \ldots, w_{i h-1}\right)$ by:

$$
\begin{gathered}
w_{1 l}=a \text { for } 1 \leq l \leq h-1, \\
w_{2 l}= \begin{cases}b & \text { if } l=h-1, \\
a & \text { elsewhere, }\end{cases}
\end{gathered}
$$




$$
\text { for } m \geq 3, w_{m l}= \begin{cases}c & \text { if } l=m-2 \\ a & \text { elsewhere }\end{cases}
$$

We have $\mathbf{W}_{1} \neq \mathbf{W}_{2}$ and for every $1 \leq i<j \leq h, \mathbf{W}_{i} \theta \mathbf{W}_{j}$ if and only if $i=1$ and $j=2$. We define the $(h-1)$-ary operation $g$ on $E_{k}$ by:

$$
g\left(x_{1}, x_{2}, \ldots, x_{h-1}\right)=\left\{\begin{array}{l}
u_{1} \text { if }\left(x_{1}, x_{2}, \ldots, x_{h-1}\right)=\mathbf{W}_{1}, \\
u_{2} \text { if }\left(x_{1}, x_{2}, \ldots, x_{h-1}\right)=\mathbf{W}_{2}, \\
u_{i} \text { if }\left(x_{1}, x_{2}, \ldots, x_{h-1}\right) \theta \mathbf{W}_{i} \text { for some } 3 \leq i \leq h-1, \\
u_{h} \text { elsewhere. }
\end{array}\right.
$$

Since $g(\theta) \subseteq\left\{\left(u_{1}, u_{2}\right) ;\left(u_{2}, u_{1}\right)\right\} \bigcup\left\{\left(u_{i}, u_{i}\right): i \in\{1 ; \cdots ; h\}\right\}, g \in \operatorname{Pol}(\theta)$.

The matrix $\left(w_{i j}\right)_{\substack{1 \leq i \leq h \\ 1 \leq j \leq h-1}}$ is a subset of $\eta^{1}$ and

$$
g\left(\left(w_{i j}\right)_{\substack{1 \leq i \leq h \\ 1 \leq j \leq h-1}}\right)=g\left(\left(\mathbf{W}_{1}\right), \ldots, g\left(\mathbf{W}_{h}\right)\right)=\left(u_{1}, \ldots, u_{h}\right) \notin \eta^{1} .
$$

Hence $g \notin \operatorname{Pol}\left(\eta^{1}\right)$. Therefore $\operatorname{Pol}\left(\eta^{1}\right) \subsetneq \operatorname{Pol}(\theta)$.

Remark 5.20. If $\operatorname{Pol}(\theta) \cap \operatorname{Pol}(\rho)$ is maximal in $\operatorname{Pol}(\theta)$, then the arity of $\rho$ is less than or equal to $t$, where $t$ is the number of equivalence classes of $\theta$. Indeed, if the arity of $\rho$ is greater than $t$, then $\eta \nsubseteq \rho$, because $\rho \neq E_{k}^{\operatorname{arity}(\rho)}$ and $\left(\eta \subseteq \rho \Rightarrow \rho=E_{k}^{\operatorname{arity}(\rho)}\right)$.

From now on we suppose that $\eta \subseteq \rho$.

According to the definition of $\rho_{0, \theta}$ we have $\rho \subseteq \rho_{0, \theta}$.

Lemma 5.21. If $\rho=\rho_{0, \theta}$, then $\operatorname{Pol}(\theta) \cap \operatorname{Pol}(\rho)$ is maximal in $\operatorname{Pol}(\theta)$. 
Proof. If $\rho=\rho_{0, \theta}$, then $\rho$ is $\theta$-closed. Hence $\rho$ is of type II and $\operatorname{Pol}(\theta) \cap \operatorname{Pol}(\rho)$ is maximal in $\operatorname{Pol}(\theta)$ from Proposition 5.13.

Now we suppose that $\rho \subsetneq \rho_{0, \theta}$ and we have two cases given by the following lemmas.

Lemma 5.22. If $\rho \subsetneq \rho_{0, \theta} \subsetneq E_{k}^{h}$, then $\operatorname{Pol}(\theta) \cap \operatorname{Pol}(\rho)$ is not maximal in $\operatorname{Pol}(\theta)$

Proof. It is easy to see that $\operatorname{Pol}(\rho) \cap \operatorname{Pol}(\theta) \subseteq \operatorname{Pol}(\theta) \cap \operatorname{Pol}\left(\rho_{0, \theta}\right) \subseteq$ $\operatorname{Pol}(\theta)$.

Let $a, b \in E_{k}$ such that $(a, b) \notin \theta$ and $\left(u_{1}, u_{2}, \ldots, u_{h}\right) \in \rho_{0, \theta} \backslash \rho$ (respectively, $E_{k}^{h} \backslash \rho$ ). Using the $h$-ary operation $f$ defined in the proof of Lemma 5.19, we show that $\operatorname{Pol}(\rho) \cap \operatorname{Pol}(\theta) \subsetneq \operatorname{Pol}(\theta) \cap \operatorname{Pol}\left(\rho_{0, \theta}\right)$ (respectively, $\left.\operatorname{Pol}(\theta) \cap \operatorname{Pol}\left(\rho_{0, \theta}\right) \subsetneq \operatorname{Pol}(\theta).\right) \quad$ Hence $\quad \operatorname{Pol}(\rho) \cap \operatorname{Pol}(\theta) \subsetneq \operatorname{Pol}(\theta) \cap \operatorname{Pol}$ $\left(\rho_{0, \theta}\right) \subsetneq \operatorname{Pol}(\theta)$.

Lemma 5.23. If $\rho \subsetneq \rho_{0, \theta}=E_{k}^{h}$ and there exists an integer $l>h$ such that $\rho_{0, \theta}^{l} \neq E_{k}^{l}$, then $\operatorname{Pol}(\theta) \cap \operatorname{Pol}(\rho)$ is not maximal in $\operatorname{Pol}(\theta)$, where $\rho_{0, \theta}^{l}$ is the relation

$$
\rho_{0, \theta}^{l}=\left\{\mathbf{u} \in E_{k}^{l} / \exists\left(e_{1}, \ldots, e_{l}\right) \in\left[u_{1}\right]_{\theta} \times \ldots \times\left[u_{l}\right]_{\theta} ;\left\{e_{1}, \ldots, e_{l}\right\}^{h} \subseteq \rho\right\}
$$

Proof. We show that $\operatorname{Pol}(\rho) \cap \operatorname{Pol}(\theta) \subsetneq \operatorname{Pol}\left(\rho_{0, \theta}^{m}\right) \cap \operatorname{Pol}(\theta) \subsetneq \operatorname{Pol}(\theta)$, where $m=\min \left\{l \in \mathbb{N} \backslash\{0 ; 1 ; 2 ; \ldots ; h\} ; \rho_{0, \theta}^{l} \neq E_{k}^{l}\right\}$. We distinguish two cases; one for each inclusion.

(i) Let us prove that $\operatorname{Pol}(\rho) \cap \operatorname{Pol}(\theta) \subsetneq \operatorname{Pol}\left(\rho_{0, \theta}^{m}\right) \cap \operatorname{Pol}(\theta)$. 
Let $f \in \mathcal{O}^{n}\left(E_{k}\right)$ be an $n$-ary operation on $E_{k}$ such that $f \in \operatorname{Pol}(\theta) \cap$ $\operatorname{Pol}(\rho)$. According to the definition of $\rho_{0, \theta}^{m}$, for

$$
\left(u_{11}, \ldots, u_{m 1}\right), \ldots,\left(u_{1 n}, \ldots, u_{m n}\right) \in \rho_{0, \theta}^{m}
$$

there exists $a_{i j} \in\left[u_{i j}\right]_{\theta}$ such that for all $j \in\{1 ; \cdots ; n\}$ we have $\left\{a_{1 j}, \ldots\right.$, $\left.a_{m j}\right\}^{h} \subseteq \rho$.

Then, it follows that there exists $a_{i j} \in\left[u_{i j}\right]_{\theta}$ such that for all $i_{1}, i_{2}$, $\ldots, i_{h} \in\{1 ; \cdots ; m\}$, we have

$$
\left(a_{i_{1} 1}, a_{i_{2} 1}, \ldots, a_{i_{h} 1}\right), \ldots,\left(a_{i_{1} n}, a_{i_{2} n}, \ldots, a_{i_{h} n}\right) \in \rho
$$

which implies that there exist $a_{i j} \in\left[u_{i j}\right]_{\theta}$ such that for all $i_{1}, i_{2}, \ldots, i_{h}$ $\in\{1 ; \cdots ; m\}$, we have

$$
\left(f\left(a_{i_{1} 1}, \ldots, a_{i_{1} n}\right), f\left(a_{i_{2} 1}, \ldots, a_{i_{2} n}\right), \ldots, f\left(a_{i_{h} 1}, \ldots, a_{i_{h} n}\right)\right) \in \rho .
$$

Since $f \in \operatorname{Pol}(\theta)$ and for all $i \in\{1 ; \cdots ; m\},\left(u_{i 1}, \ldots, u_{i n}\right) \theta\left(a_{i 1}, \ldots, a_{i n}\right)$, it appears that

$$
\left(f\left(u_{11}, \ldots, u_{1 n}\right), \ldots, f\left(u_{m 1}, \ldots, u_{m n}\right)\right) \in \rho_{0, \theta}^{m} \quad \text { and } \quad f \in \rho_{0, \theta}^{m} .
$$

Hence $f \in \operatorname{Pol}\left(\rho_{0, \theta}^{m}\right) \cap \operatorname{Pol}(\theta)$.

Let $\left(v_{1}, \ldots, v_{h}\right) \in E_{k}^{h} \backslash \rho$ and $(a, b) \notin \theta$, using the $h$-ary operation $f$ on $E_{k}$, specified by: 


$$
f\left(x_{1}, x_{2}, \ldots, x_{h}\right)=\left\{\begin{array}{l}
v_{1} \text { if }\left(x_{1}, x_{2}, \ldots, x_{h}\right) \theta(a, a, \ldots, a), \\
v_{2} \text { if }\left(x_{1}, x_{2}, \ldots, x_{h}\right) \theta(a, b, a, \ldots, a), \\
\vdots \\
v_{h} \text { if }\left(x_{1}, x_{2}, \ldots, x_{h}\right) \theta(a, a, \ldots, a, b, a), \\
v_{h} \text { if }\left(x_{1}, x_{2}, \ldots, x_{h}\right) \theta(a, a, \ldots, a, a, b), \\
v_{1} \text { otherwise, }
\end{array}\right.
$$

and the fact that $m>h$ and $\rho_{0, \theta}^{m}$ is totally reflexive, we obtain $f \notin \operatorname{Pol}(\rho)$ and $f \in \operatorname{Pol}\left(\rho_{0, \theta}^{m}\right)$.

(ii) We prove that $\operatorname{Pol}\left(\rho_{0, \theta}^{m}\right) \cap \operatorname{Pol}(\theta) \subsetneq \operatorname{Pol}(\theta)$.

Let $\left(u_{1}, \ldots, u_{m}\right) \in E_{k}^{m} \backslash \rho_{0, \theta}^{m}\left(E_{k}^{m} \backslash \rho_{0, \theta}^{m}\right.$ is not empty because $\left.\rho_{0, \theta}^{m} \neq E_{k}^{m}\right)$ and $(a, b) \notin \theta$. Let us consider the $m$-ary operation $h$ on $E_{k}$ defined by:

$$
h\left(x_{1}, \ldots, x_{m}\right)=\left\{\begin{array}{l}
u_{1} \text { if }\left(x_{1}, \ldots, x_{m}\right) \theta(a, \ldots, a, a)=a_{1}, \\
u_{2} \text { if }\left(x_{1}, \ldots, x_{m}\right) \theta(a, b, a, \ldots, a)=a_{2}, \\
u_{3} \text { if }\left(x_{1}, \ldots, x_{m}\right) \theta(a, a, b, a, \ldots, a)=a_{3}, \\
\vdots \\
u_{m} \text { if }\left(x_{1}, \ldots, x_{m}\right) \theta(a, a, a, \ldots, a, b)=a_{m}, \\
u_{m} \text { otherwise. }
\end{array}\right.
$$

The implication $a_{i} \theta a_{j} \Rightarrow i=j$ allows us to say that $h \in \operatorname{Pol}(\theta)$. It is clear that 


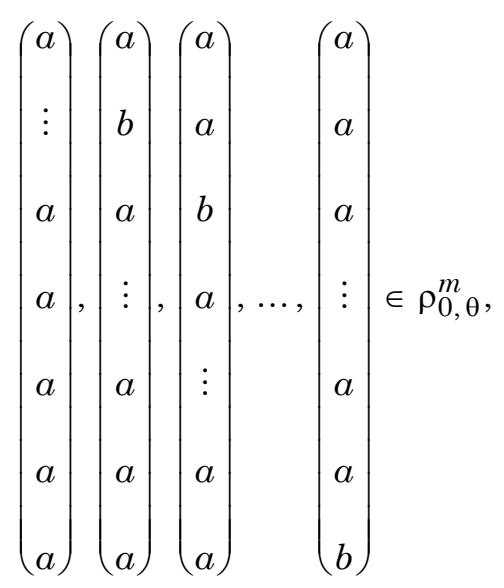

but following the definition of $h$, it appears that

$$
\left(u_{1}, u_{2}, u_{3}, \ldots, u_{m}\right)=\left(h\left(a_{1}\right), h\left(a_{2}\right), h\left(a_{3}\right), \ldots, h\left(a_{m}\right) \notin \rho_{0, \theta}^{m} .\right.
$$

Therefore, $h \notin \operatorname{Pol}\left(\rho_{0, \theta}^{m}\right)$ and $\operatorname{Pol}\left(\rho_{0, \theta}^{m}\right) \cap \operatorname{Pol}(\theta) \subsetneq \operatorname{Pol}(\theta)$.

Hence $\operatorname{Pol}(\rho) \cap \operatorname{Pol}(\theta) \subsetneq \operatorname{Pol}\left(\rho_{0, \theta}^{m}\right) \cap \operatorname{Pol}(\theta) \subsetneq \operatorname{Pol}(\theta)$.

In the light of Lemma 5.23, it is natural to suppose $\rho_{0, \theta}=E_{k}^{h}$ and $\rho_{0, \theta}^{l}=E_{k}^{l}$ for all $l>h$. In particular, for $l=t$, we have $\rho_{0, \theta}^{t}=E_{k}^{t}$ and there exists $\left(x_{1}, x_{2}, \ldots, x_{t}\right) \in C_{0} \times C_{1} \times \ldots \times C_{t-1}$ such that $\left\{x_{1}, x_{2}, \ldots\right.$, $\left.x_{t}\right\}^{h} \subseteq \rho$. Let $\varsigma$ be the relation $\varsigma=\left\{\mathbf{a} \in E_{k}^{h} \mid \exists u_{i j} \in\left[a_{i}\right]_{\theta}, 1 \leq i, j \leq h\right.$ with $i \neq j$ such that $\forall j \in\{1 ; \cdots ; h\}$, $\left(a_{j}, u_{j_{1} j}, u_{j_{2} j}, \ldots, u_{j_{h-1} j}\right) \in \rho$, with $\left.\left\{j_{1} ; \cdots ; j_{h-1}\right\}=\{1 ; \cdots ; h\} \backslash\{j\}\right\}$.

Since $\rho$ is totally symmetric, $\varsigma=\bigcap_{\sigma \in \mathcal{S}_{h}}\left(\rho_{1, \theta}\right)_{\sigma}$ and it is obvious that $\rho \subseteq \varsigma$

Lemma 5.24. If $\rho=\varsigma$, then $\operatorname{Pol}(\theta) \cap \operatorname{Pol}(\rho)$ is maximal in $\operatorname{Pol}(\theta)$. 
Proof. If $\rho=\varsigma$, then $\rho$ is weakly $\theta$-closed of order 1 . Hence $\rho$ is a relation of type III. By Proposition 5.13, $\operatorname{Pol}(\theta) \cap \operatorname{Pol}(\rho)$ is maximal in $\operatorname{Pol}(\theta)$.

Lemma 5.25. If $\rho \subsetneq \varsigma \subsetneq E_{k}^{h}$ then $\operatorname{Pol}(\theta) \cap \operatorname{Pol}(\rho)$ is not maximal in $\operatorname{Pol}(\theta)$

Proof. We prove that $\operatorname{Pol}(\rho) \cap \operatorname{Pol}(\theta) \subsetneq \operatorname{Pol}(\theta) \cap \operatorname{Pol}(\varsigma) \subsetneq \operatorname{Pol}(\theta)$. Let $f \in \operatorname{Pol}(\theta) \cap \operatorname{Pol}(\rho), n$-ary.

Let $\left(a_{11}, a_{21}, \ldots, a_{h 1}\right), \ldots,\left(a_{1 n}, a_{2 n}, \ldots, a_{s n}\right) \in \varsigma$, we show that

$$
\left(f\left(a_{11}, \ldots, a_{1 n}\right), f\left(a_{21}, \ldots, a_{2 n}\right), \ldots, f\left(a_{h 1}, \ldots, a_{h n}\right)\right) \in \varsigma .
$$

Firstly, we show that $\left(f\left(a_{11}, \ldots, a_{1 n}\right), \ldots, f\left(a_{h 1}, \ldots, a_{h n}\right)\right) \in \rho_{1, \theta}$. Since $\left(a_{1 i}, \ldots, a_{h i}\right) \in \varsigma$ for $1 \leq i \leq n$, there exist $u_{j i} \in\left[a_{j i}\right]_{\theta}, 2 \leq j \leq h$ such that $\left(a_{1 i}, u_{2 i}, \ldots, u_{h i}\right) \in \rho$ for $1 \leq i \leq n$. Therefore

$$
\left(f\left(a_{11}, \ldots, a_{1 n}\right), f\left(u_{21}, \ldots, u_{2 n}\right), \ldots, f\left(u_{h 1}, \ldots, u_{h n}\right)\right) \in \rho,
$$

and $f\left(u_{j 1}, \ldots, u_{j n}\right) \theta f\left(a_{j 1}, \ldots, a_{j n}\right)$ for $2 \leq j \leq n$. Hence

$$
\left(f\left(a_{11}, \ldots, a_{1 n}\right), f\left(a_{21}, \ldots, a_{2 n}\right), \ldots, f\left(a_{h 1}, \ldots, a_{h n}\right)\right) \in \rho_{1, \theta} .
$$

Secondly, we show that $\left(f\left(a_{11}, \ldots, a_{1 n}\right), \ldots, f\left(a_{h 1}, \ldots, a_{h n}\right)\right) \in\left(\rho_{1, \theta}\right)_{\sigma}$ for $\sigma \in \mathcal{S}_{h}$. Let $\sigma \in \mathcal{S}_{h}$. Since $\left(a_{1 i}, \ldots, a_{h i}\right) \in\left(\rho_{1, \theta}\right)_{\sigma}$ for $1 \leq i \leq n$, it follows that $\left(a_{\sigma-1(1) i}, \ldots, a_{\sigma-1(h) i}\right) \in \rho_{1, \theta}$ for $1 \leq i \leq n$. Hence

$$
\left(f\left(a_{\sigma-1(1) 1}, \ldots, a_{\sigma-1(1) n}\right), \ldots, f\left(a_{\sigma-1(h) 1}, \ldots, a_{\sigma-1(h) n}\right)\right) \in \rho_{1, \theta} .
$$

Therefore $\left(f\left(a_{11}, \ldots, a_{1 n}\right), \ldots, f\left(a_{h 1}, \ldots, a_{h n}\right)\right) \in\left(\rho_{1, \theta}\right)_{\sigma}$.

Thus $\left(f\left(a_{11}, \ldots, a_{1 n}\right), \ldots, f\left(a_{h 1}, \ldots, a_{h n}\right)\right) \in \varsigma$.

And it follows that $\operatorname{Pol}(\rho) \cap \operatorname{Pol}(\theta) \subseteq \operatorname{Pol}(\theta) \cap \operatorname{Pol}(\varsigma)$. 
As $\rho \subsetneq \varsigma$ we have $\varsigma \backslash \rho \neq \emptyset$; then with $\left(u_{1}, u_{2}, \ldots, u_{h}\right) \in \varsigma \backslash \rho$ and $(a, b) \notin \theta$ fixed; using the $h$-ary operation $f$ defined by (5.1), and the same argument used in the proof of Lemma 5.22, it follows that $f \in \operatorname{Pol}(\varsigma)$ and $f \notin \operatorname{Pol}(\rho)$. Then $\operatorname{Pol}(\rho) \cap \operatorname{Pol}(\theta) \subsetneq \operatorname{Pol}(\theta) \cap \operatorname{Pol}(\varsigma)$.

It is obvious to see that $\operatorname{Pol}(\theta) \cap \operatorname{Pol}(\varsigma) \subsetneq \operatorname{Pol}(\theta)$ (because $\varsigma \neq E_{k}^{h}$ ).

Let us take a fixed element $\left(u_{1}, u_{2}, \ldots, u_{h}\right) \in E_{k}^{h} \backslash \varsigma$ and $(a, b) \notin \theta$. Using the operation $f$ defined above, we easily obtain $f \in \operatorname{Pol}(\theta)$.

On the other hand

$$
\left(\begin{array}{llllll}
a & a & a & \cdots & a & a \\
a & b & a & \cdots & a & a \\
& & \cdots & \cdots & & \\
a & a & \cdots & a & b & a \\
a & a & \cdots & a & a & b
\end{array}\right) \subseteq \varsigma,
$$

but by the definition of $f$ we have $\left(u_{1}, u_{2}, \ldots, u_{h}\right)=(f(a, a, \ldots, a), f(a, b, a, \ldots, a), \ldots, f(a, a, \ldots, a, a, b)) \notin \varsigma$. It follows that $f \notin \operatorname{Pol}(\varsigma)$; thus $\operatorname{Pol}(\rho) \cap \operatorname{Pol}(\theta) \subsetneq \operatorname{Pol}(\varsigma) \cap \operatorname{Pol}(\theta) \subsetneq \operatorname{Pol}(\theta)$.

Lemma 5.26. If $\rho \subsetneq \varsigma=E_{k}^{h}$ and there exists $h<l \leq t$ with $\varsigma^{l} \neq E_{k}^{l}$, then $\operatorname{Pol}(\theta) \cap \operatorname{Pol}(\rho)$ is not maximal in $\operatorname{Pol}(\theta)$, where $\varsigma^{l}=\bigcap_{\sigma \in \mathcal{S}_{h}}\left(\rho_{1, \theta}^{l}\right)_{\sigma}$ with

$$
\rho_{1, \theta}^{l}=\left\{\left(a_{1}, \ldots, a_{l}\right) \in E_{k}^{l} / \exists u_{i} \in\left[a_{i}\right]_{\theta}, 2 \leq i \leq l:\left\{a_{1} ; u_{2}, u_{3}, \ldots, u_{l}\right\}^{h} \subseteq \rho\right\} .
$$


Proof. We set $m:=\min \left\{l \in\{0 ; 1 ; \cdots ; t\} \backslash\{0 ; 1 ; 2 ; \ldots ; h\} / \varsigma^{l} \neq E_{k}^{l}\right\}$. We want to prove that $\operatorname{Pol}(\rho) \cap \operatorname{Pol}(\theta) \subsetneq \operatorname{Pol}\left(\varsigma^{m}\right) \cap \operatorname{Pol}(\theta) \subsetneq \operatorname{Pol}(\theta)$.

We use the similar argument as in the proof of Lemma 5.25 to show that $\operatorname{Pol}(\rho) \cap \operatorname{Pol}(\theta) \subseteq \operatorname{Pol}\left(\varsigma^{m}\right) \cap \operatorname{Pol}(\theta)$.

Let $\left(u_{1}, u_{2}, \ldots, u_{h}\right) \in \varsigma \backslash \rho$ and $(a, b) \notin \theta$ fixed.

Using the $h$-ary operation $f$ defined by (5.1), we have again $f \notin \operatorname{Pol}(\rho)$ and $f \in \operatorname{Pol}\left(\varsigma^{m}\right) ;$ since $\left(u_{1}, u_{2}, \ldots, u_{h}\right) \in E_{k}^{h}=\varsigma$, there exist $a_{i j} \in\left[u_{i}\right]_{\theta}$, $i, j \in\{1, \ldots, s\}$ with $i \neq j$ such that for all $j \in\{1, \ldots, h\}$,

$$
\left(u_{j}, a_{j_{1} j}, a_{j_{2} j}, \ldots, a_{j_{h-1} j}\right) \in \rho
$$

with $\left\{j_{1}, \ldots, j_{h-1}\right\}=\{1, \ldots, h\} \backslash\{j\}$. Then it follows that $\left\{u_{1}, u_{2}, \ldots\right.$, $\left.u_{h}\right\}^{m} \subseteq \varsigma^{m}$. Moreover, as we have $\varsigma^{m} \neq E_{k}^{m}$, there exists $\left(u_{1}, \ldots, u_{m}\right)$ $\in E_{k}^{m} \backslash \varsigma^{m}$. Given $\left(u_{1}, \ldots, u_{m}\right) \in E_{k}^{m} \backslash \varsigma^{m}$ and $(a, b) \notin \theta$.

Using the $m$-ary operation $h$ defined by (5.2), and the same argument used in the proof of Lemma 5.23, we have $h \in \operatorname{Pol}(\theta)$ but $h \notin \operatorname{Pol}\left(\varsigma^{m}\right)$. Thus $\operatorname{Pol}(\rho) \cap \operatorname{Pol}(\theta) \subsetneq \operatorname{Pol}\left(\varsigma^{m}\right) \cap \operatorname{Pol}(\theta) \subsetneq \operatorname{Pol}(\theta)$.

The previous lemma suggests to suppose that for each $l \in\{h ; \cdots ; t\}$, $\varsigma^{l}=E_{k}^{l}$.

Let $m=\max \left\{\left|C_{i}\right| ; 0 \leq i \leq t-1\right\}$ and we denote by $\gamma^{\prime}$ the relation $\gamma^{\prime}=\left\{\left(a_{1}, \ldots, a_{m}, a_{m+1}, \ldots, a_{m+t-1}\right) \in E_{k}^{m+t-1} / \forall\{i ; j\} \subseteq\{1 ; \cdots ; m\}, a_{i} \theta a_{j} ;\right.$ $\exists u_{i} \in\left[a_{i}\right]_{\theta}, m+1 \leq i \leq m+t-1, \forall 1 \leq i \leq m$, $\left.\left\{a_{i} ; u_{m+1} ; \ldots ; u_{m+t-1}\right\}^{h} \subseteq \rho\right\}$. 
We have $\operatorname{Pol}\left(\gamma^{\prime}\right) \subseteq \operatorname{Pol}(\theta)$. We define two relations $\epsilon_{=}$and $\epsilon_{\theta}^{\prime}$ on $\{1 ; \cdots ; m+t-1\}$ by:

$(i, j) \in \epsilon_{=} \Leftrightarrow i=j$ and $(i, j) \in \epsilon_{\theta}^{\prime} \Leftrightarrow(i=j$ or $\{i, j\} \subseteq\{1 ; \ldots ; m\})$,

$\epsilon_{=}$and $\epsilon_{\theta}^{\prime}$ are equivalence relations and $\gamma^{\prime} \subseteq D_{\epsilon_{=} \epsilon_{\theta}^{\prime}}$.

Lemma 5.27. If $\forall l \in\{h, h+1, \ldots, t\}, \varsigma^{l}=E_{k}^{h}$ and $\gamma^{\prime} \neq D_{\epsilon_{=} \epsilon_{\theta}^{\prime}}$, then $\operatorname{Pol}(\theta) \cap \operatorname{Pol}(\rho)$ is not maximal in $\operatorname{Pol}(\theta)$.

Proof. We just have to prove that $\operatorname{Pol}(\rho) \cap \operatorname{Pol}(\theta) \subsetneq \operatorname{Pol}\left(\gamma^{\prime}\right) \subsetneq \operatorname{Pol}(\theta)$.

Let $f \in \mathcal{O}^{n}\left(E_{k}\right)$ be an $n$-ary operation on $E_{k}$ such that $f \in \operatorname{Pol}(\theta) \cap$ $\operatorname{Pol}(\rho)$. Let

$$
\left(a_{11}, \ldots, a_{m+t-11}\right), \ldots,\left(a_{1 n}, \ldots, a_{m+t-1 n}\right) \in \gamma^{\prime} .
$$

According to the definition of $\gamma^{\prime}$, for all $1 \leq j \leq n$, for all $\alpha, \beta \in\{1 ; \cdots ; m\}$, we have $\left[a_{\alpha j}\right]_{\theta}=\left[a_{\beta j}\right]_{\theta}$ and for all $j \in\{1 ; \cdots ; n\}$, there exists $u_{r j} \in\left[a_{r j}\right]_{\theta}$ with $r \in\{m+1 ; \cdots ; m+t-1\}$ such that for all $i \in\{1 ; \cdots ; m\}$,

$$
\left\{a_{i j} ; u_{m+1 j} ; u_{m+2 j} ; \ldots ; u_{m+t-1 j}\right\}^{h} \subseteq \rho .
$$

Since $f \in \operatorname{Pol}(\theta) \cap \operatorname{Pol}(\rho)$, for all $\alpha, \beta \in\{1 ; \cdots ; m\},\left[f\left(a_{\alpha 1}, \ldots, a_{\alpha n}\right)\right]_{\theta}=$ $\left[f\left(a_{\beta 1}, \ldots, a_{\beta n}\right)\right]_{\theta}$ and for all $r \in\{m+1 ; \cdots ; m+t-1\}, f\left(u_{r 1}, \ldots, u_{r n}\right) \in$ $\left[f\left(a_{r 1}, \ldots, a_{r n}\right)\right]_{\theta}$. It follows that for all $i \in\{1 ; \cdots ; m\}$

$$
\left\{f\left(a_{i 1}, \ldots, a_{i n}\right) ; f\left(u_{m+11}, \ldots, u_{m+1 n}\right) ; \ldots ; f\left(u_{m+t-11}, \ldots, u_{m+t-1 n}\right)\right\}^{h} \subseteq \rho .
$$

Consequently,

$$
\left(f\left(a_{11}, \ldots, a_{1 n}\right), \ldots, f\left(a_{m+t-11}, \ldots, a_{m+t-1 n}\right)\right) \in \gamma^{\prime},
$$

and then $f \in \operatorname{Pol}\left(\gamma^{\prime}\right)$. 
Let $\left(u_{1}, u_{2}, \ldots, u_{h}\right) \in E_{k}^{h} \backslash \rho$ and $(a, b) \notin \theta$ fixed. Using the $h$-ary operation $f$ defined by (5.1), we have $f \notin \operatorname{Pol}(\rho)$ and $f \in \operatorname{Pol}\left(\gamma^{\prime}\right)$; since $\left(u_{1}, u_{2}, \ldots, u_{h}\right) \in E_{k}^{h}=\varsigma$, it follows that there exist $a_{i j} \in\left[u_{i}\right]_{\theta}$; $i, j \in\{1 ; \cdots ; h\}$ with $i \neq j$ such that for all $j \in\{1 ; \cdots ; h\}$,

$$
\left(u_{j}, a_{j_{1} j}, a_{j_{2} j}, \ldots, a_{j_{h-1} j}\right) \in \rho
$$

with $\left\{j_{1}, \ldots, j_{h-1}\right\}=\{1 ; \cdots ; h\} \backslash\{j\}$. It follows that

$$
\bigcup_{i \in\{1 ; \cdots ; h\}}\left\{u_{i}\right\}^{m} \times\left\{u_{l} ; l \in\{1 ; \cdots ; h\} \backslash\{i\}\right\}^{t-1} \subseteq \gamma^{\prime} .
$$

From the fact that $\gamma^{\prime} \neq D_{\epsilon_{=} \epsilon_{\theta}^{\prime}}$, we deduce that $D_{\epsilon_{=} \epsilon_{\theta}^{\prime}} \backslash \gamma^{\prime} \neq \emptyset$.

Given $\left(v_{1}, \ldots, v_{m+t-1}\right) \in D_{\epsilon_{=} \epsilon_{\theta}^{\prime}} \backslash \gamma^{\prime},(a, b) \notin \theta$ and consider the following $(m+t-1)$-ary operation defined on $E_{k}$ by:

$$
h\left(x_{1}, \ldots, x_{m+t-1}\right)= \begin{cases}v_{1} & \text { if }\left(x_{1}, \ldots, x_{m+t-1}\right) \theta(a, \ldots, a, a)=a_{1}, \\ v_{m+1} & \text { if }\left(x_{1}, \ldots, x_{m+t-1}\right) \theta(a, b, a, \ldots, a)=a_{2}, \\ v_{m+2} & \text { if }\left(x_{1}, \ldots, x_{m+t-1}\right) \theta(a, a, b, a \ldots, a)=a_{3}, \\ & \vdots \\ v_{m+t-1} & \text { if }\left(x_{1}, \ldots, x_{m+t-1}\right) \theta(a, a, a, \ldots a, b)=a_{t}, \\ v_{m+t-1} & \text { otherwise. }\end{cases}
$$

Since $a_{i} \theta a_{j} \Rightarrow i=j, h \in \operatorname{Pol}(\theta)$. From the fact that $\{a, b\}^{h} \subseteq \rho$ we have $h \notin \operatorname{Pol}\left(\gamma^{\prime}\right)$. Indeed, 


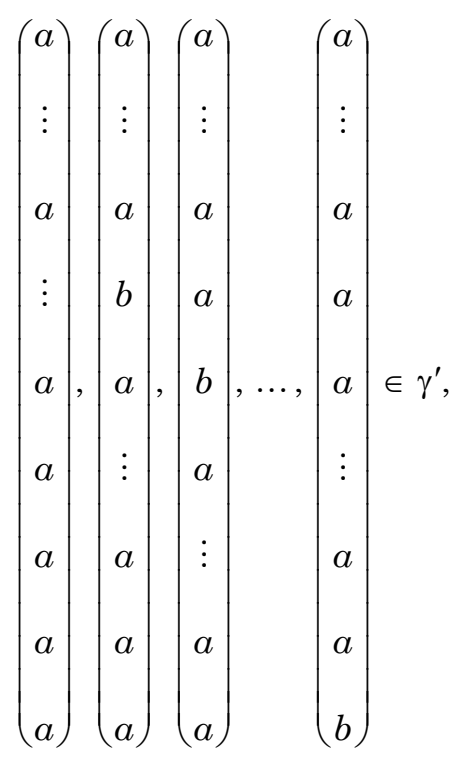

but

$$
\left(v_{1}, \ldots, v_{1}, v_{m+1}, \ldots, v_{m+t-1}\right)=\left(h\left(a_{1}\right), \ldots, h\left(a_{1}\right), h\left(a_{2}\right), \ldots, h\left(a_{t}\right)\right) \notin \gamma^{\prime} .
$$

In the next step, we assume that

$$
\gamma^{\prime}=D_{\epsilon=\epsilon_{\theta}^{\prime}},
$$

i.e., for all $i \in\{0 ; \cdots ; t-1\}$, there exists $u_{j i} \in C_{j}, j \in\{0 ; \cdots ; t-1\} \backslash\{i\}$ such that for all $a \in C_{i},\left\{a ; u_{1 i} ; \ldots ; u_{i-1 i}, u_{i+1 i} ; \ldots ; u_{t i}\right\}^{h} \subseteq \rho$. Let $\zeta$ be the relation defined by:

$$
\begin{aligned}
& \zeta=\left\{\mathbf{a} \in E_{k}^{h} \mid \exists u_{i} \in\left[a_{i}\right]_{\theta}, \text { such that }\left(a_{i}, u_{i_{1}}, \ldots, u_{i_{h-1}}\right) \in \rho, i \in\{1 ; \cdots ; h\},\right. \\
&\text { and } \left.\left\{i_{1} ; \ldots ; i_{h-1}\right\}=\{1 ; \cdots ; h\} \backslash\{i\}\right\} .
\end{aligned}
$$

Our goal is to show that if $\operatorname{Pol}(\theta) \cap \operatorname{Pol}(\rho)$ is maximal in $\operatorname{Pol}(\theta)$, then $\zeta=E_{k}^{h}$. Before that, let us prove the following result. 
Lemma 5.28. If $\rho=\zeta$, then $\rho=E_{k}^{h}$.

Proof. We suppose that $\rho=\zeta$. Let $a=\left(a_{1}, a_{2}, \ldots, a_{s}\right) \in E_{k}^{h}$. If there exists $\{i ; j\} \subseteq\{1 ; \cdots ; h\}$ with $i \neq j$ such that $\left(a_{i}, a_{j}\right) \in \theta$, then $a \in \rho$ since $\eta \subseteq \rho$. Else, according to (5.3), for all $i \in\{1, \ldots, t\}$, there exist $u_{j i} \in C_{j}, j \in\{0 ; \cdots ; t-1\} \backslash\{i\}$ such that for all $b \in C_{i}$,

$$
\left\{b ; u_{0 i} ; \ldots ; u_{i-1 i}, u_{i+1 i} ; \ldots ; u_{t-1 i}\right\}^{h} \subseteq \rho,
$$

where $C_{j}=\left[a_{j}\right]_{\theta}, j \in\{1 ; \cdots ; h\}$. Hence

$$
\left(C_{i} \cup\left\{u_{0 i}, \ldots ; u_{t-1 i}\right\} \backslash\left\{u_{i i}\right\}\right)^{h} \subseteq \rho, 0 \leq i \leq t-1 .
$$

Therefore

$$
\left(a_{1}, a_{2}, \ldots, a_{h}\right) \in \zeta=\rho
$$

It follows that $\rho=E_{k}^{h}$.

From Lemma 5.28 and the fact that $\rho \neq E_{k}^{h}$, we obtain $\rho \subsetneq \zeta \subseteq E_{k}^{h}$. Now we can prove the following lemma.

Lemma 5.29. If $\zeta \neq E_{k}^{h}$, then $\operatorname{Pol}(\theta) \cap \operatorname{Pol}(\rho)$ is not maximal in $\operatorname{Pol}(\theta)$.

Proof. We have to prove that $\operatorname{Pol}(\rho) \cap \operatorname{Pol}(\theta) \subsetneq \operatorname{Pol}(\theta) \cap \operatorname{Pol}(\zeta) \subsetneq \operatorname{Pol}(\theta)$. Let $f \in \mathcal{O}^{n}\left(E_{k}\right)$ be an $n$-ary operation on $E_{k}$ such that $f \in \operatorname{Pol}(\theta) \cap \operatorname{Pol}(\rho)$. Let

$$
\left(a_{11}, a_{21}, \ldots, a_{h 1}\right), \ldots,\left(a_{1 n}, a_{2 n}, \ldots, a_{h n}\right) \in \zeta .
$$

From the definition of $\zeta$, for all $i \in\{1 ; \cdots ; h\}$, for all $j \in\{1, \ldots, n\}$, there exist $u_{i j} \in\left[a_{i j}\right]_{\theta}$ such that

$$
\left(a_{1 j}, u_{2 j}, \ldots, u_{h j}\right),\left(u_{1 j}, a_{2 j}, \ldots, u_{h j}\right), \ldots,\left(u_{1 j}, u_{2 j}, \ldots, u_{(h-1) j}, a_{h j}\right) \in \rho
$$

and as $f \in \operatorname{Pol}(\rho)$ we have

$$
\left(f\left(a_{11}, \ldots, a_{1 n}\right), f\left(u_{21}, \ldots, u_{2 n}\right), \ldots, f\left(u_{h 1}, \ldots, u_{h n}\right)\right) \in \rho,
$$




$$
\begin{gathered}
\left(f\left(u_{11}, \ldots, u_{1 n}\right), f\left(a_{21}, \ldots, a_{2 n}\right), f\left(u_{31}, \ldots, u_{3 n}\right), \ldots, f\left(u_{h 1}, \ldots, u_{h n}\right)\right) \in \rho, \ldots \\
\left(f\left(u_{11}, \ldots, u_{1 n}\right), \ldots, f\left(u_{(s-1) 1}, \ldots, u_{(h-1) n}\right), f\left(a_{h 1}, \ldots, a_{h n}\right)\right) \in \rho .
\end{gathered}
$$

Moreover, as $f \in \operatorname{Pol}(\theta)$, we have $f\left(u_{i 1}, \ldots, u_{i n}\right) \theta f\left(a_{i 1}, \ldots, a_{i n}\right)$; therefore, using the definition of $\zeta$,

$$
\left(f\left(a_{11}, \ldots, a_{1 n}\right), f\left(a_{21}, \ldots, a_{2 n}\right), \ldots, f\left(a_{h 1}, \ldots, a_{h n}\right)\right) \in \zeta .
$$

Then $\operatorname{Pol}(\rho) \cap \operatorname{Pol}(\theta) \subseteq \operatorname{Pol}(\theta) \cap \operatorname{Pol}(\zeta)$.

As $\rho \subsetneq \zeta$ we have $\zeta \backslash \rho$ is not empty. Given $\left(u_{1}, u_{2}, \ldots, u_{h}\right) \in \zeta \backslash \rho$ and $(a, b) \notin \theta$, using the $h$-ary operation $f$ defined by (5.1), and the same argument used in the proof of Lemma 5.22, we obtain $f \in \operatorname{Pol}(\zeta)$ and $f \notin \operatorname{Pol}(\rho)$. Therefore, $\operatorname{Pol}(\rho) \cap \operatorname{Pol}(\theta) \subsetneq \operatorname{Pol}(\theta) \cap \operatorname{Pol}(\zeta)$. It is obvious that $\operatorname{Pol}(\theta) \cap \operatorname{Pol}(\zeta) \subsetneq \operatorname{Pol}(\theta)$ (because $\left.\zeta \neq E_{k}^{h}\right)$. Given $\left(u_{1}, u_{2}, \ldots, u_{h}\right) \in E_{k}^{h} \backslash \zeta$ and $\quad(a, b) \notin \theta . f \in \operatorname{Pol}(\theta) \quad$ and $\quad f \notin \operatorname{Pol}(\zeta) ; \quad$ thus $\operatorname{Pol}(\rho) \cap \operatorname{Pol}(\theta) \subsetneq$ $\operatorname{Pol}(\zeta) \cap \operatorname{Pol}(\theta) \subsetneq \operatorname{Pol}(\theta)$

Lemma 5.30. If $\zeta=E_{k}^{h}$ and $\zeta^{k} \neq E_{k}^{k}$, then $\operatorname{Pol}(\theta) \cap \operatorname{Pol}(\rho)$ is not maximal in $\operatorname{Pol}(\theta)$, where $\zeta^{l}$ is the l-ary relation on $E_{k}$ specified by

$$
\begin{array}{r}
\zeta^{l}=\left\{\left(a_{1}, \ldots, a_{l}\right) \in E_{k}^{l} / \exists u_{i} \in\left[a_{i}\right]_{\theta} / \forall i \in\{1 ; \cdots ; l\},\right. \\
\left.\left\{u_{1} ; \ldots ; u_{i-1} ; a_{i} ; u_{i+1} ; \ldots ; u_{l}\right\}^{h} \subseteq \rho\right\} .
\end{array}
$$

Proof. We prove that $\operatorname{Pol}(\rho) \cap \operatorname{Pol}(\theta) \subsetneq \operatorname{Pol}\left(\zeta^{k}\right) \subsetneq \operatorname{Pol}(\theta)$.

It is easy to see that $\operatorname{Pol}(\rho) \cap \operatorname{Pol}(\theta) \subseteq \operatorname{Pol}\left(\zeta^{k}\right)$.

Given $\left(u_{1}, u_{2}, \ldots, u_{h}\right) \in E_{k}^{h} \backslash \rho$ and $(a, b) \notin \theta$, using the $h$-ary operation $f$ defined by (5.1), we have again $f \notin \operatorname{Pol}(\rho)$ and $f \in \operatorname{Pol}\left(\zeta^{k}\right)$; indeed, since $\left(u_{1}, u_{2}, \ldots, u_{h}\right) \in E_{k}^{h}=\zeta$, there exist $v_{i} \in\left[u_{i}\right]_{\theta}$, 
$i \in\{1 ; \cdots ; h\}$ such that for all $i \in\{1 ; \cdots ; h\},\left\{u_{i}, v_{i_{1}}, \ldots, v_{i_{h-1}}\right\}^{h} \subseteq \rho$, with $\left\{i_{1}, i_{2}, \ldots, i_{h-1}\right\}=\{1 ; \cdots ; h\} \backslash\{i\}$, and it follows that $\left\{u_{1}, u_{2}, \ldots, u_{h}\right\}^{k} \subseteq \zeta^{k}$.

Moreover, as we have $\zeta^{k} \neq E_{k}^{k}$, then $E_{k}^{k} \backslash \zeta^{k} \neq \emptyset$. Given $\left(w_{1}, \ldots\right.$, $\left.w_{k}\right) \in E_{k}^{k} \backslash \zeta^{k},(a, b) \notin \theta$, using the $k$-ary operation $h$ defined on $E_{k}$, by:

$$
h\left(x_{1}, \ldots, x_{k}\right)=\left\{\begin{aligned}
w_{1} & \text { if }\left(x_{1}, \ldots, x_{k}\right) \theta(a, \ldots, a, a)=a_{1}, \\
w_{2} & \text { if }\left(x_{1}, \ldots, x_{k}\right) \theta(a, b, a, \ldots, a)=a_{2}, \\
w_{3} & \text { if }\left(x_{1}, \ldots, x_{k}\right) \theta(a, a, b, a, \ldots, a)=a_{3}, \\
& \vdots \\
w_{k} & \text { if }\left(x_{1}, \ldots, x_{k}\right) \theta(a, a, a, \ldots, a, b)=a_{k}, \\
w_{k} & \text { otherwise }
\end{aligned}\right.
$$

and the same argument used for the similar operation $h$ in the proof of Lemma 5.23 yields $h \in \operatorname{Pol}(\theta)$ and $h \notin \operatorname{Pol}\left(\zeta^{k}\right)$.

Thus $\operatorname{Pol}(\rho) \cap \operatorname{Pol}(\theta) \subsetneq \operatorname{Pol}\left(\zeta^{k}\right) \cap \operatorname{Pol}(\theta) \subsetneq \operatorname{Pol}(\theta)$.

Now, we continue our induction process with the assumption $\varsigma^{l}=E_{k}^{l}$ for all $h \leq l \leq t$ and $\zeta^{k}=E_{k}^{k}$. Since $(0, \ldots, k-1) \in E_{k}^{k}$, there exist $u_{i} \in[i]_{\theta}$ such that

$$
\left\{u_{0} ; u_{1} ; \ldots ; u_{i-1} ; i ; u_{i+1} ; \ldots ; u_{k-1}\right\}^{h} \subseteq \rho
$$

for all $i \in E_{k}$. We set

$$
T_{i}:=\min \left(C_{i}\right) \cap\left\{u_{0} ; u_{1} ; \ldots ; u_{k-1}\right\},
$$

for all $i \in E_{k}$. Therefore $\left\{T_{0} ; \cdots ; T_{t-1}\right\}$ is a transversal of order 1 . 
Before the main induction, we define the sequence $\left({ }^{h} \beta_{n}\right)$ by: ${ }^{h} \beta_{0}=\eta$, $h_{\beta_{1}}=\rho_{0, \theta},{ }^{h} \beta_{2}=\varsigma$, and for $l \geq 3$,

$$
{ }^{h} \beta_{l}=\bigcap_{\sigma \in \mathcal{S}_{h}}\left(\rho_{l-1, \theta}\right)_{\sigma} .
$$

Let $n \in\{1 ; \cdots ; h-1\}$ and assume that there exists a transversal $T$ of order $n-1$ for the $\theta$-classes. Set $T=\left\{u_{0} ; u_{1} ; \ldots ; u_{t-1}\right\}$; then for every $a_{1}, a_{2}, \ldots a_{n-1} \in E_{k},\left\{a_{1} ; a_{2} ; \cdots a_{n-1} ; u_{0} ; u_{1} ; \cdots ; u_{t-1}\right\}^{h} \subseteq \rho$ and $\rho \subseteq{ }^{h} \beta_{n+1}$.

Lemma 5.31. If $\rho={ }^{h} \beta_{n+1} \subsetneq E_{k}^{h}$, then $\operatorname{Pol}(\theta) \cap \operatorname{Pol}(\rho)$ is maximal in $\operatorname{Pol}(\theta)$.

Proof. If $\rho={ }^{h} \beta_{n+1} \subsetneq E_{k}^{h}$, then $\rho$ is weakly $\theta$-closed of order $n$. Hence $\rho$ is a relation of type III. Using Proposition 5.13 $\operatorname{Pol}(\theta) \cap \operatorname{Pol}(\rho)$ is maximal in $\operatorname{Pol}(\theta)$.

Lemma 5.32. If $\rho \subsetneq^{h} \beta_{n+1} \subsetneq E_{k}^{h}$, then $\operatorname{Pol}(\theta) \cap \operatorname{Pol}(\rho)$ is not maximal in $\operatorname{Pol}(\theta)$.

Proof. We have to prove that $\operatorname{Pol}(\rho) \cap \operatorname{Pol}(\theta) \subsetneq \operatorname{Pol}(\theta) \cap \operatorname{Pol}\left({ }^{h} \beta_{n+1}\right) \subsetneq$ $\operatorname{Pol}(\theta)$. It is easy to show that $\operatorname{Pol}(\rho) \cap \operatorname{Pol}(\theta) \subseteq \operatorname{Pol}(\theta) \cap \operatorname{Pol}\left({ }^{h} \beta_{n+1}\right)$.

Using the $h$-ary operation $f$ defined by (5.1), we show that $\operatorname{Pol}(\rho) \cap$ $\operatorname{Pol}(\theta) \subsetneq \operatorname{Pol}\left({ }^{h} \beta_{n+1}\right) \cap \operatorname{Pol}(\theta) \subsetneq \operatorname{Pol}(\theta)$.

Lemma 5.33. If $\rho \subsetneq{ }^{h} \beta_{n+1}=E_{k}^{h}$ and ${ }^{h} \beta_{n+1}^{l} \neq E_{k}^{l}$ for some $l>h$, then $\operatorname{Pol}(\theta) \cap \operatorname{Pol}(\rho)$ is not maximal in $\operatorname{Pol}(\theta)$; where ${ }^{h} \beta_{n+1}^{l}=\bigcap_{\sigma \in \mathcal{S}_{l}}\left(\rho_{n, \theta}^{l}\right)_{\sigma}$ with

$$
\begin{array}{r}
\rho_{n, \theta}^{l}:=\left\{\left(a_{1}, \ldots, a_{l}\right) \in E_{k}^{l} / \exists u_{i} \in\left[a_{i}\right]_{\theta}, n+1 \leq i \leq l:\right. \\
\left.\left\{a_{1} ; a_{2} ; \cdots ; a_{n} ; u_{n+1}, \ldots, u_{l}\right\}^{h} \subseteq \rho\right\} .
\end{array}
$$


Proof. Denote $m:=\min \left\{l \in \mathbb{N} \backslash\{0 ; 1 ; 2 ; \ldots ; h\} /^{h} \beta_{n+1}^{l} \neq E_{k}^{l}\right\}$. Before proving that $\operatorname{Pol}(\rho) \cap \operatorname{Pol}(\theta) \subsetneq \operatorname{Pol}\left({ }^{h} \beta_{n+1}^{m}\right) \cap \operatorname{Pol}(\theta) \subsetneq \operatorname{Pol}(\theta)$, we first prove that $\operatorname{Pol}(\rho) \cap \operatorname{Pol}(\theta) \subseteq \operatorname{Pol}\left({ }^{h} \beta_{n+1}^{m}\right) \cap \operatorname{Pol}(\theta)$.

Let $f \in \operatorname{Pol}(\theta) \cap \operatorname{Pol}(\rho)$ be an $p$-ary operation on $E_{k}$. Let

$$
\left(a_{11}, \ldots, a_{m 1}\right), \ldots,\left(a_{1 p}, \ldots, a_{m p}\right) \in{ }^{h} \beta_{n+1}^{m} .
$$

By definition of ${ }^{h} \beta_{n+1}^{m}$, for all $c \in\{1 ; \cdots ; p\}$, for all $\sigma \in \mathcal{S}_{m}$, there exist $u_{\sigma(r) c} \in\left[a_{\sigma(r) c}\right]_{\theta}, n+1 \leq r \leq m$ such that

$$
\left\{a_{\sigma(1) c} ; \ldots ; a_{\sigma(n) c} ; u_{\sigma(n+1) c} ; \ldots ; u_{\sigma(m) c}\right\}^{h} \subseteq \rho,
$$

and from the fact that $f \in \operatorname{Pol}(\rho)$ it follows that for all $\sigma \in \mathcal{S}_{m}$,

$$
\begin{gathered}
\left\{f\left(a_{\sigma(1) 1}, \ldots, a_{\sigma(1) p}\right) ; \ldots ; f\left(a_{\sigma(n) 1}, \ldots, a_{\sigma(n) p}\right) ;\right. \\
\left.f\left(u_{\sigma(n+1) 1}, \ldots, u_{\sigma(n+1) p}\right) ; \ldots ; f\left(u_{\sigma(m) 1}, \ldots, u_{\sigma(m) p}\right)\right\}^{h} \subseteq \rho .
\end{gathered}
$$

Since $f \in \operatorname{Pol}(\theta)$, it follows that for all $i \in\{n+1 ; \cdots ; m\}$, and for all $d \in\{n+1 ; \cdots ; m\}$,

$$
\sigma(d)=i \Rightarrow f\left(u_{\sigma(d) 1}, u_{\sigma(d) 2}, \ldots, u_{\sigma(d) p}\right) \theta f\left(a_{i 1}, \ldots, a_{i p}\right) .
$$

From the definition of ${ }^{h} \beta_{n+1}^{m}$, it follows that

$$
\left(f\left(a_{11}, \ldots, a_{1 p}\right), \ldots, f\left(a_{m 1}, \ldots, a_{m p}\right)\right) \in{ }^{h} \beta_{n+1}^{m} .
$$

Then $f \in \operatorname{Pol}\left({ }^{h} \beta_{n+1}^{m}\right) \cap \operatorname{Pol}(\theta)$.

Fix $\left(v_{1}, \ldots, v_{h}\right) \in E_{k}^{h} \backslash \rho$ and let $(a, b) \notin \theta$. Using the $h$-ary operation $f$ defined by (5.1)(where we replace $u_{i}$ by $v_{i}, 1 \leq i \leq h$ ), we have $f \notin \operatorname{Pol}(\rho)$ and $f \in \operatorname{Pol}\left({ }^{h} \beta_{n+1}^{m}\right)$. Indeed, due to the fact that

$$
\left(v_{1}, \ldots, v_{h}\right) \in E_{k}^{h}={ }^{h} \beta_{n+1},
$$


for all $\sigma \in \mathcal{S}_{h}$ there exist $d_{r} \in\left[v_{r}\right]_{\theta}, n+1 \leq r \leq h$ such that,

$$
\left\{v_{\sigma(1)} ; \ldots ; a_{\sigma(n)} ; d_{\sigma(n+1)} ; \ldots ; d_{\sigma(h)}\right\}^{h} \subseteq \rho
$$

Therefore, it follows that $\left\{v_{1} ; v_{2} ; \ldots ; v_{h}\right\}^{m} \subseteq{ }^{h} \beta_{n+1}^{m}$.

Moreover, as we have ${ }^{h} \beta_{n+1}^{m} \neq E_{k}^{m}$, then $E_{k}^{m} \backslash h_{\beta} m$ is not an empty relation. Given $\left(v_{1}, \ldots, v_{m}\right) \in E_{k}^{m} \backslash h_{\beta_{n+1}^{m}}$ and $(a, b) \notin \theta$; using the $m$-ary operation $h$ defined by (5.2) (where we replace $u_{i}$ by $v_{i}$, $1 \leq i \leq m$ ), and the same argument used in the proof of Lemma 5.23, it follows that $h \in \operatorname{Pol}(\theta)$ and $h \notin \operatorname{Pol}\left({ }^{h} \beta_{n+1}^{m}\right)$. Thus $\operatorname{Pol}(\rho) \cap \operatorname{Pol}(\theta) \subsetneq \operatorname{Pol}$ $\left({ }^{h} \beta_{n+1}^{m}\right) \cap \operatorname{Pol}(\theta) \subsetneq \operatorname{Pol}(\theta)$.

It is natural now to suppose that for each $l \in\{h ; \cdots ; t\},{ }^{h} \beta_{n+1}^{l}=E_{k}^{l}$.

Let $m_{1}, \ldots, m_{n}$ be integers such that $m_{1}>m_{2}>\cdots>m_{n}$ and for each $i \in\{0 ; \cdots ; t-1\},\left|C_{i}\right| \leq \min \left\{m_{1}, \ldots, m_{n}\right\}$ or $\left|C_{i}\right| \in\left\{m_{1}, \ldots, m_{n}\right\}$. We set $m=m_{1}+\ldots+m_{n}$. In this part we will use these notations

$$
\begin{aligned}
& \mathbf{a}:=\left(a_{1}, \ldots, a_{m_{1}}, a_{m_{1}+1}, \ldots, a_{m_{1}+m_{2}}, \ldots, a_{m_{1}+\ldots+m_{n}}, a_{m+1}, \ldots, a_{m+t-n}\right), \\
& \prod=\left\{1 ; \cdots ; m_{1}\right\} \times\left\{m_{1}+1 ; \cdots ; m_{1}+m_{2}\right\} \times \ldots \times\left\{m_{1}+\cdots+m_{n-1}+1 ; \cdots ; m\right\},
\end{aligned}
$$

and

$$
\begin{aligned}
\prod^{\prime}=\left\{1 ; \cdots ; m_{1}\right\}^{2} \cup\left\{m_{1}+1 ; \cdots ; m_{1}+m_{2}\right\}^{2} \cup \ldots \cup\left\{m_{1}+\ldots\right. & +m_{n-1} \\
& +1 ; \cdots ; m\}^{2} .
\end{aligned}
$$

Let ${ }^{h} \beta_{n+1}^{\prime}$ be the relation

$$
\begin{aligned}
h_{\beta_{n+1}^{\prime}}= & \left\{\mathbf{a} \in E_{k}^{m+t-n} / \forall(i, j) \in \prod^{\prime}, a_{i} \theta a_{j} ;\right. \\
& \exists u_{i} \in\left[a_{i}\right]_{\theta}, m+1 \leq i \leq m+t-n, \text { such that }
\end{aligned}
$$




$$
\left.\forall\left(i_{1}, \ldots, i_{n}\right) \in \prod,\left\{a_{i_{1}}, \ldots, a_{i_{n}}, u_{m+1}, \ldots, u_{m+t-n}\right\}^{h} \subseteq \rho\right\} .
$$

We have $\operatorname{Pol}\left({ }^{h} \beta_{n+1}^{\prime}\right) \subseteq \operatorname{Pol}(\theta)$.

We define two relations $\varrho_{1}$ and $\varrho_{2}$ on $\{1 ; \cdots ; m+t-n\}$ by:

$$
(i, j) \in \varrho_{1} \Leftrightarrow i=j \text { and }(i, j) \in \varrho_{2} \Leftrightarrow\left(i=j \text { or }(i, j) \in \prod^{\prime}\right),
$$

$\varrho_{1}$ and $\varrho_{2}$ are equivalence relations and ${ }^{h} \beta_{n+1}^{\prime} \subseteq D_{\varrho_{1} \varrho_{2}}$.

Lemma 5.34. If $\forall l \in\{h, h+1, \ldots, t\},{ }^{h} \beta_{n+1}^{l}=E_{k}^{h}$ and ${ }^{h} \beta_{n+1}^{\prime} \neq D_{\varrho_{1} \varrho_{2}}$, then $\operatorname{Pol}(\theta) \cap \operatorname{Pol}(\rho)$ is not maximal in $\operatorname{Pol}(\theta)$.

Proof. It is similar to the proof of Lemma 5.27.

In what follows, we assume that

$$
h_{\beta_{n+1}^{\prime}} \neq D_{\varrho_{1} \varrho_{2}},
$$

i.e., for all $i_{1}, \ldots, i_{n} \in\{0 ; \cdots ; t-1\}$, there exist $u_{j I} \in C_{j}, j \in\{0 ; \cdots ; t-1\}$ $\backslash\left\{i_{1}, \ldots, i_{n}\right\}$, with $I=\left\{i_{1}, \ldots, i_{n}\right\}$ such that for all $a_{i_{r}} \in C_{i_{r}}, r \in\{1 ; \cdots ; n\}$ we have

$$
\left\{a_{i_{1}} ; \cdots ; a_{i_{n}}, u_{1 I} ; \ldots ; u_{t-n I}\right\}^{h} \subseteq \rho .
$$

We obtain a transversal of order $n$ for the $\theta$-classes. Hence we can continue the previous induction until

$$
{ }^{h} \beta_{h}=\bigcap_{\sigma \in \mathcal{S}_{h}}\left(\rho_{h-1, \theta}\right)_{\sigma}=E_{k}^{h} .
$$

From now on, we suppose that ${ }^{h} \beta_{h}=E_{k}^{h}$.

Lemma 5.35. If ${ }^{h} \beta_{h}=E_{k}^{h}$ and there exists $l>h$ such that ${ }^{h} \beta_{h}^{l} \neq E_{k}^{h}$, then $\operatorname{Pol}(\rho) \cap \operatorname{Pol}(\theta)$ is not maximal in $\operatorname{Pol}(\theta)$. 
Proof. Set $m=\min \left\{l \in\{1 ; \cdots ; k\} \backslash\{1 ; \cdots ; h\} /{ }^{h} \beta_{h}^{l} \neq E_{k}^{h}\right\}$. It is easy to prove that $\operatorname{Pol}(\rho) \cap \operatorname{Pol}(\theta) \subsetneq \operatorname{Pol}\left({ }^{h} \beta_{h}^{m}\right) \cap \operatorname{Pol}(\theta) \subsetneq \operatorname{Pol}(\theta)$.

Lemma 5.36. If ${ }^{h} \beta_{h}^{k}=E_{k}^{k}$, then $\operatorname{Pol}(\theta) \cap \operatorname{Pol}(\rho)$ is maximal in $\operatorname{Pol}(\theta)$.

Proof. If ${ }^{h} \beta_{h}^{k}=E_{k}^{k}$, then each equivalence class of $\theta$ has a central element of $\rho$. Since $\eta \subseteq \rho, \rho$ is of type $\mathrm{I}$ and $\operatorname{Pol}(\rho) \cap \operatorname{Pol}(\theta)$ is maximal in $\operatorname{Pol}(\theta)$ by Proposition 5.13.

We are ready now to give the proof of Proposition 5.18 and Theorem 5.9.

Proof of Proposition 5.18. Combining Lemmas 5.19, 5.22, 5.23, $5.25,5.26,5.27,5.29,5.30,5.32,5.33,5.35,5.36$ and Remark 5.20, we obtain the result.

Proof of Theorem 5.9. It follows from Propositions 5.13 and 5.18.

We investigate the case of $h$-regular relations.

5.2. $h$-regular relations. As an $h$-regular relation is totally reflexive and totally symmetric, some results stated in the previous subsection can apply. Besides an hregular generated relation does not contain a central element. We prove that there is submaximality if and only if $\rho$ is $\theta$-closed (or of type II). We begin this subsection with some examples of $h$-regular relations.

Example 5.37. Let $k \geq 3$ be an integer and $0 \leq i<j<r<n \leq k-1$, we denote by $A_{i, j, r}$ and $A_{i, j, r, n}$ the sets

$$
A_{i, j, r}:=\left\{(\sigma(i), \sigma(j), \sigma(r)) ; \sigma \in \mathcal{S}_{\{i ; j ; r\}}\right\},
$$

and

$$
A_{i, j, r, n}:=\left\{(\sigma(i), \sigma(j), \sigma(r), \sigma(n)) ; \sigma \in \mathcal{S}_{\{i ; j ; r ; n\}}\right\}
$$


We consider the following equivalence relations $\theta_{6} \theta_{7} \theta_{8}$ on $E_{12}$ defined respectively by their equivalence classes denoted by $C_{m}^{i}, 6 \leq i \leq 8$ as follows:

$$
\begin{gathered}
C_{0}^{6}=\{0 ; 1 ; 2 ; 3 ; 4\}, C_{1}^{6}=\{5 ; 6 ; 7\}, C_{2}^{6}=\{8 ; 9 ; 10 ; 11\} ; \\
C_{0}^{7}=\{0 ; 1 ; 5 ; 8\}, C_{1}^{7}=\{2 ; 6 ; 9 ; 11\}, C_{2}^{7}=\{3 ; 4 ; 7 ; 10\} ; \\
C_{0}^{8}=\{0 ; 1\}, C_{1}^{8}=\{2\}, C_{2}^{8}=\{3 ; 4\}, C_{3}^{8}=\{5\}, C_{4}^{8}=\{6\}, C_{5}^{8}=\{7\}, \\
C_{6}^{8}=\{8\}, C_{7}^{8}=\{9\}, C_{8}^{8}=\{10\}, C_{9}^{8}=\{11\} ;
\end{gathered}
$$

and the relation

$$
\begin{gathered}
\Upsilon_{6}=\left\{\left(x_{1}, x_{2}, x_{3}\right) \in E_{12}^{3}:\left(x_{1}, x_{2}\right) \in \theta_{6} \text {, or }\left(x_{2}, x_{3}\right) \in \theta_{6}, \text { or }\left(x_{1}, x_{3}\right) \in \theta_{6}\right\}, \\
\Upsilon_{7}=\bigcup_{\sigma \in \mathcal{S}_{3}}(\Upsilon)_{\sigma}
\end{gathered}
$$

where

$$
\Upsilon=\left\{\left(x_{1}, x_{2}, x_{3}\right) \in E_{12}^{3}:\left(x_{1}, x_{2}\right) \in \theta_{6},\left(x_{2}, x_{3}\right) \in \theta_{7}\right\} .
$$

It is easy to see that $\Upsilon_{6}$ is a $\theta_{6}$-closed 3-regular relation associated to $T=\left\{\theta_{6}\right\}$, and $\Upsilon_{7}$ is a $\theta_{8}$-closed 3-regular relation associated to $T=\left\{\theta_{6} ; \theta_{7}\right\}$.

We continue with the characterization of $\theta$-closed $h$-regular relation.

Lemma 5.38. For $h \geq 3$, let $\rho$ be an h-regular relation on $E_{k}$ determined by the h-regular family $T=\left\{\theta_{1} ; \cdots ; \theta_{m}\right\}$ and $\theta$ be a nontrivial equivalence relation on $E_{k} . \rho$ is $\theta$-closed iff $\theta \subseteq \theta_{i}$, for all $1 \leq i \leq m$. 
Proof. $\Rightarrow$ Firstly, we show that $\eta=\left\{\left(a_{1}, \ldots, a_{h}\right) \in E_{k}^{h}:\left(a_{1}, a_{2}\right) \in \theta\right\} \subseteq \rho$. Let $\left(a_{1}, \ldots, a_{h}\right) \in \eta$; since $\rho$ is totally reflexive we have $\left(a_{1}, a_{1}, a_{3}, \ldots, a_{h}\right) \in \rho$; we also have $\left(a_{1}, a_{2}, a_{3}, \ldots, a_{h}\right) \theta\left(a_{1}, a_{1}, a_{3}, \ldots, a_{h}\right)$. Hence $\left(a_{1}, \ldots, a_{h}\right) \in \rho_{0, \theta}=\rho$. Our next step is to show that $\theta \subseteq \theta_{i}$ for all $\quad 1 \leq i \leq m$. Let $\quad i \in\{1, \ldots, m\} \quad$ and $\quad(a, b) \in \theta ; \quad$ set $A_{i}=E_{k} / \theta_{i} \backslash\left\{[a]_{\theta_{i}},[b]_{\theta_{i}}\right\}$. It is easy to see that $\left|A_{i}\right| \geq h-2$; choose $\left(a_{1}, \ldots, a_{h-2}\right) \in E_{k}^{h-2}$ such that $\left[a_{p}\right]_{\theta_{i}} \in A_{i}$ for all $1 \leq p \leq h-2$ and $\left(a_{p}, a_{q}\right) \notin \theta_{i}$ for all $1 \leq p<q \leq h-2$. Due to $\eta \subseteq \rho$, we have $\left(a, b, a_{1}, \ldots, a_{h-2}\right) \in \rho$; therefore $(a, b) \in \theta$ and $\theta \subseteq \theta_{i}$.

$\Leftarrow$ It follows from the fact that $\theta \circ \theta_{i} \circ \theta \subseteq \theta_{i}$ for all $1 \leq i \leq m$.

Lemma 5.39. For $h \geq 3$, let $\rho$ be an $h$-ary relation and $\theta$ be a nontrivial equivalence relation on $E_{k}$. $\rho$ is a $\theta$-closed $h$-regular relation iff there exists an h-regular relation $\psi$ on $E_{t}$ such that $\rho=\varphi^{-1}(\psi)$.

Proof. Firstly, let us assume that there exists an $h$-regular relation $\psi$ on $E_{t}$ and put $\perp=\left\{v_{1} ; \cdots ; v_{n}\right\}$ the $h$-regular family associated to $\psi$. Clearly $\varphi^{-1}(\perp)=\left\{\varphi^{-1}\left(\nu_{1}\right) ; \cdots ; \varphi^{-1}\left(\nu_{n}\right)\right\}$ is an $h$-regular family and $\rho=\varphi^{-1}(\psi)$ is exactly the $h$-regular relation associated to $\varphi^{-1}(\perp)$. Moreover, for all $1 \leq i \leq n$ we have $\theta \subseteq \varphi^{-1}\left(\nu_{i}\right)$. Therefore, by Lemma 5.38, $\rho$ is $\theta$-closed.

Conversely, assume that $\rho$ is an $h$-regular relation determined by the $h$-regular family $T=\left\{\theta_{1} ; \cdots ; \theta_{r}\right\}$ and $\rho$ is $\theta$-closed. Clearly, for all $1 \leq i \leq r, \varphi\left(\theta_{i}\right)$ is an equivalence relation with exactly $h$ equivalence classes whose are the images of equivalence classes of $\theta_{i}$ by $\varphi$. It follows that $\bigcap\left\{\varphi\left(B_{i}\right) \mid 1 \leq i \leq r\right\}$ is non-empty for arbitrary equivalence classes $\varphi\left(B_{i}\right)$ of $\varphi\left(\theta_{i}\right), 1 \leq i \leq r$. Therefore $\varphi(T)=\left\{\varphi\left(\theta_{1}\right) ; \cdots ; \varphi\left(\theta_{r}\right)\right\} \quad$ is an 
$h$-regular family and $\varphi(\rho)$ is an $h$-regular relation on $E_{t}$, associated to $\varphi(T)$. Moreover $\varphi^{-1}(\varphi(\rho))=\rho$ because $\rho$ is $\theta$-closed. Therefore we have the result.

We end this subsection with the characterization of $h$-regular relations $\rho$ such that $\operatorname{Pol}(\theta) \cap \operatorname{Pol}(\rho)$ is maximal in $\operatorname{Pol}(\theta)$. We need the next lemma to prove our main result.

Lemma 5.40 ([7]). Let $\chi \subseteq E_{t}^{h}$ such that $\operatorname{Pol}(\chi)$ is maximal in $\mathcal{L}_{t}$. Then $\operatorname{Pol}(\theta) \cap \operatorname{Pol}\left(\varphi^{-1}(\chi)\right)$ is submaximal in $\operatorname{Pol}(\theta)$.

Proof. See the proof of Lemma 18.2.5, page 565 in [7].

Lemma 5.41. If $\operatorname{Pol}(\rho) \cap \operatorname{Pol}(\theta)$ is maximal in $\operatorname{Pol}(\theta)$, then $\eta \subseteq \rho$.

Proof. See the proof of Lemma 5.19.

Now we give the result of this subsection.

Proposition 5.42. Let $\theta$ be a nontrivial equivalence relation and $\rho$ be an h-regular relation on $E_{k}$ determined by the h-regular family $T=\left\{\theta_{1} ; \cdots ; \theta_{m}\right\} . \operatorname{Pol}(\theta) \cap \operatorname{Pol}(\rho)$ is maximal in $\operatorname{Pol}(\theta)$ if and only if $\rho$ is $\theta$-closed.

Proof. $\Rightarrow$ It follows from Lemma 5.41 that $\eta \subseteq \rho$; and in the light of the proof of Lemma 5.38, we conclude that $\theta \subseteq \theta_{i}$ for all $1 \leq i \leq m$. It follows from Lemma 5.38 that $\rho$ is $\theta$-closed.

$\Leftarrow$ Combining Lemmas 5.38, 5.39, 5.40, we have the result.

\section{Conclusion}

In this paper, we characterized the relations $\rho$ from the Rosenberg's list for which $\operatorname{Pol}(\theta) \cap \operatorname{Pol}(\rho)$ is maximal in $\operatorname{Pol}(\theta)$, where $\theta$ is a nontrivial equivalence relation. The classification of all central relations 
$\rho$ on $E_{k}$ such that the clone $\operatorname{Pol}(\theta) \cap \operatorname{Pol}(\rho)$ is maximal in $\operatorname{Pol}(\theta)$ improves Temgoua and Rosenberg's results [18]. We plan in a future project to characterize the meet-irreducible submaximal clones of $\operatorname{Pol}(\theta)$ for a nontrivial equivalence relation $\theta$.

\section{References}

[1] K. A. Baker and A. F. Pixley, Polynomial interPolation and the Chinese remainder theorem for algebraic systems, Math. Z. 143 (1975), 165-174.

[2] S. Burris and H. P. Sankappanavar, A Course in Universal Algebra, SpringerVerlag, 1981.

[3] K. Denecke and W. L. Wismath, Universal Algebra and Applications in Theoretical Computer Science, Chapman \& Hall / CRC, 2002.

[4] A. Grecianu, Clones sous-maximaux inf-réductibles. Mémoire numérisé par la Division de la gestion de documents et des archives de l'Université de Montréal, 2009.

$$
\text { http://hdl.handle.net/1866/7887 }
$$

[5] S. V. Jablonskij, Functional constructions in many-valued logics, Tr. Mat. Inst. Steklova 51 (1958), 5-142 (in Russian).

[6] B. Larose, Maximal subclones of the clone of isotone operations on a bounded finite order, C. R. Acad. Sci. Paris 314 (1992), 245-247.

[7] D. Lau, Function Algebras on Finite Sets, Springer, 2006.

[8] M. Pinsker, Rosenberg's Characterization of Maximal Clones, Diploma Thesis, Vienna Univ. of Technology, 2002.

[9] R. Poeschel, Concrete representation of algebriac structure and a general Galois theory, Contribution to General Algebra, (Proc. Klagenfurt Conf., Klagenfurt, 1978), Verlag J. Heyn, Klagenfurt, 1 (1979), 249-272.

[10] M. Ponjanie, On Traces of Maximal Clones, Master Thesis, Univ. of Novi Sad, 2003.

[11] E. L. Post, Introduction to a general theory of elementary propositions, Amer. J. Math. 43 (1921), 163-185.

[12] E. L. Post, The Two-valued Iterative Systems of Mathematical Logic, Ann. Math. Studies, Vol. 5, Princeton University Press, 1941.

[13] R. W. Quackenbush, A new proof of Rosenberg's primal algebra characterization theorem. In: Finite algebra and multiple valued logic (Szeged, 1979), Colloq. Math. Soc. J. Bolyai, North Holland, Amsterdam 28 (1981), 603-634. 
[14] I. G. Rosenberg, La structure des fonctions de plusieurs variables sur un ensemble fini, C. R. Acad. Sci. Paris, Ser. A-B, 260 (1965), 3817-3819.

[15] I. G. Rosenberg, Functional completeness in many-valued logics; the structure of functions of several variables on finite sets, Rozpravy, Československ Akad. Věd Řada Mat. Přírod. Věd 80 (1970), 3-93 (in German)

[16] I. G. Rosenberg, ber die functionals Vollständigkeit in dem mehrwertigen Logiken, Rozpravy Ceskoslovenké Akad. ved. Ser. Math. Nat. Sci. 80 (1970), 3-93.

[17] I. G. Rosenberg and A. Szendrei, Submaximal clones with a prime order automorphism, Acta Sci. Math. 49 (1985), 29-48.

[18] E. R. A. Temgoua and I. G. Rosenberg, Binary central relations and submaximal clones determined by nontrivial equivalence relations, Algebra Universalis, Springer Basel AG, 67 (2012), 299-311.

[19] E. R. A. Temgoua, Meet-irreducible submaximal clones determined by nontrivial equivalence relations, Algebra Universalis 70 (2013), 175-196. 